\title{
Beyond black-boxes in Bayesian inverse problems and model validation: applications in solid mechanics of elastography
}

\author{
Lukas Bruder \\ Technical University of Munich \\ Phaedon-Stelios Koutsourelakis \\ Technical University of Munich
}

March 8, 2018

\begin{abstract}
The present paper is motivated by one of the most fundamental challenges in inverse problems, that of quantifying model discrepancies and errors. While significant strides have been made in calibrating model parameters, the overwhelming majority of pertinent methods is based on the assumption of a perfect model. Motivated by problems in solid mechanics which, as all problems in continuum thermodynamics, are described by conservation laws and phenomenological constitutive closures, we argue that in order to quantify model uncertainty in a physically meaningful manner, one should break open the black-box forward model. In particular we propose formulating an undirected probabilistic model that explicitly accounts for the governing equations and their validity. This recasts the solution of both forward and inverse problems as probabilistic inference tasks where the problem's state variables should not only be compatible with the data but also with the governing equations as well. Even though the probability densities involved do not contain any black-box terms, they live in much higher-dimensional spaces. In combination with the intractability of the normalization constant of the undirected model employed, this poses significant challenges which we propose to address with a linearlyscaling, double-layer of Stochastic Variational Inference. We demonstrate the capabilities and efficacy of the proposed model in synthetic forward and inverse problems (with and without model error) in elastography.
\end{abstract}

Keywords: Uncertainty quantification; Variational inference; Inverse problems; Model error; Stochastic optimization; Bayesian modeling 


\section{Contents}

1 Introduction 3

2 Proposed modeling framework $\quad 7$

2.1 Augmented prior/posterior densities . . . . . . . . . . . . . . 9

2.2 Representation of the unknown state variables . . . . . . . . . . 13

2.3 Rephrasing forward/inverse problems as probabilistic inference tasks . 15

3 Variational Inference $\quad 19$

3.1 VI for forward problems . . . . . . . . . . . . . . . . . 19

3.2 VI for inverse problems . . . . . . . . . . . . . . . . 20

3.3 Form of approximating densities . . . . . . . . . . . . . . . . . . . . . . . . . . . . . 22

3.4 Stochastic Variational Inference . . . . . . . . . . . . . . . 23

4 Numerical illustrations $\quad 25$

4.1 Comparison of diagonal, banded and full covariance approximations . 26

4.2 Forward problem . . . . . . . . . . . . . . . . . 28

4.3 Inverse problem without model error . . . . . . . . . . . . . . . 29

4.4 Inverse problem with model error . . . . . . . . . . . . . 30

5 Conclusion $\quad 43$

A Appendix $\quad 53$

A.1 Derivatives of $\mathcal{L}_{\text {for }} \ldots \ldots \ldots \ldots \ldots \ldots$

A.2 Derivatives of $\mathcal{L}_{i n v} \ldots \ldots \ldots \ldots \ldots$. . . . . . . . . . . . . . . . . . . . . . . . . . . . . . . . 53

A.3 Parameter update using Adam . . . . . . . . . . . . . 54 


\section{Introduction}

The extensive use of large-scale computational models poses several challenges in model calibration and validation $[8,27]$. Traditionally in numerical simulations, the emphasis has been placed on decreasing the truncation/discretization errors. Nevertheless, the fidelity of the predictions of such simulations depends strongly on assigning proper values to the model parameters as well as utilizing high-fidelity models. This in turn necessitates a data-driven approach where elaborate computational models are fused with data, originating either from experiments/measurements or from models of higher fidelity (e.g. molecular dynamics). This process is naturally fraught with significant uncertainties. One such source is obviously the noise in the data which constitutes probabilistic estimates more rational. This is particularly important when multiple hypotheses are consistent with the data or the level of confidence in the estimates produced needs to be quantified. Another source of uncertainty, which is largely unaccounted for, is model uncertainty [37, 50, 79]. Namely, the parameters which are calibrated are associated with a particular forward model (in our case a system of (discretized) PDEs consisting of conservation laws and constitutive equations or closures) but one cannot be certain about the validity of the model employed. In general, there will be deviations between the physical reality where measurements are made, and the idealized mathematical/computational description.

An application that motivates this work comes from biomechanics and the identification of the mechanical properties of biological materials, in the context of non-invasive medical diagnosis (elastography). While in certain cases mechanical properties can also be measured directly by excising multiple tissue samples, non-invasive procedures offer obvious advantages in terms of ease, cost and reducing the risk of complications to the patient. Rather than x-ray techniques which capture variations in density, the identification of stiffness, or mechanical properties in general, can potentially lead to earlier and more accurate diagnosis [42, 78], provide valuable insights that differentiate between modalities of the same pathology [28], monitor the progress of treatments and ultimately lead to patient-specific treatment strategies.

All elastographic techniques consist of the following three basic steps [36] : 1) excite the tissue using a (quasi-)static, harmonic or transient source, 2) (indirectly) measure tissue deformation (e.g. displacements, velocities) using an imaging technique such as ultrasound [82], magnetic resonance [74] or optical tomography [58], and 3) infer the mechanical properties from this data using a suitable continuum mechanical model of the tissue's deformation. Indirect or iterative or model-based methods for solving the latter problem (in contrast to direct methods [3]) admit an inverse problem formulation where the discrepancy [44]) between observed and model-predicted deformations is minimized with respect to the material fields of interest $[1,35,36,76,81]$. While these approaches utilize directly the raw data, they generally imply an increased computational cost as the forward problem, and potentially parametric derivatives, have to be solved/computed several times. This effort is amplified when stochastic/statistical formulations are employed as those arising from the Bayesian paradigm. 
The solution of such model calibration (and validation) problems in the Bayesian framework is hampered by two main difficulties. The first pertains to their computational efficiency and stems from the poor scaling of traditional Bayesian inference tools with respect to the dimensionality of the unknown parameter vector - another instance of the curse-of-dimensionality. In elastography, the model parameters of interest (i.e. material properties) exhibit spatial variability which requires fine-discretization in order to be captured. This variability can also span different scales [38, 62]. Standard Markov Chain Monte Carlo (MCMC, [46]) techniques require an exorbitant number of likelihood evaluations (i.e. solutions of the forward model) in order to reach convergence $[70,85,88,89]$. As each of these calls implies the solution of very large systems of (non)linear and potentially transient, equations, it is critical to minimize their number particularly in time-sensitive applications. Advanced sampling schemes, involving adaptive MCMC [20, 52, 65] and Sequential Monte Carlo (SMC, [29, 62, 71]), exploit the physical insight and the use of multi-fidelity solvers in order to expedite the inference process. Nevertheless, they fail to address fundamental challenges as the number of forward calls can still be in the order of tens of thousands. Several attempts have also been directed towards using emulators, surrogates or reduced-order models of various kinds $[10,16,33,64,69,90]$ but such a task is severely hindered by the input dimensionality. The use of first and second-order derivatives has also been advocated either in a standard MCMC format or by developing advanced sampling strategies. These are generally available by solving appropriate adjoint problems which are wellunderstood in the context of deterministic formulations [15, 40, 43, 68, 84]. More recent treatments, attempt to exploit the (potentially) lower intrinsic dimensionality of the target posterior by identifying subspaces where either most of the probability mass is contained [41] or where maximal sensitivity is observed [24, 25, 26, 95]. This enables inference tasks to be performed on much lower dimensions which are not hampered by the aforementioned difficulties. Generally all such schemes construct such approximations around the MAP point by employing local information (e.g. gradients) and therefore are not suitable for multi-modal or highly non-Gaussian posteriors.

The second challenge facing Bayesian inverse problems pertains to the model structure itself. This is especially important in the context of biomechanics applications where inferring model parameters associated with an incorrect model can lead to incorrect diagnosis and prognosis ${ }^{1}$. Despite progress in Bayesian model calibration tasks [50], the issue of model validation still poses many open questions, which, if not adequately addressed, can lead to biased and over-confident parameter estimates and predictions [14]. Most efforts have been directed towards providing quantitative comparisons between competing models. In the latter context, Bayes factors [56] provide a rigorous means of model comparison. Nevertheless, apart from the computational difficulties involved, they do not reveal the key driver behind model uncertainty nor do they quantify its effect on the predictions of the model. Alternatively, the most widely-

\footnotetext{
1"I remember my friend Johnny von Neumann used to say, "With four parameters I can fit an elephant and with five I can make him wiggle his trunk." A meeting with Enrico Fermi, Nature 427, $297 ; 2004$.
} 


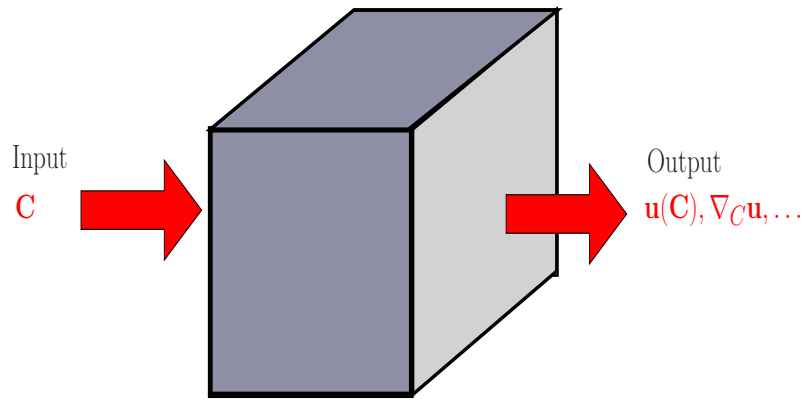

Figure 1: Black-box setting for Bayesian model calibration.

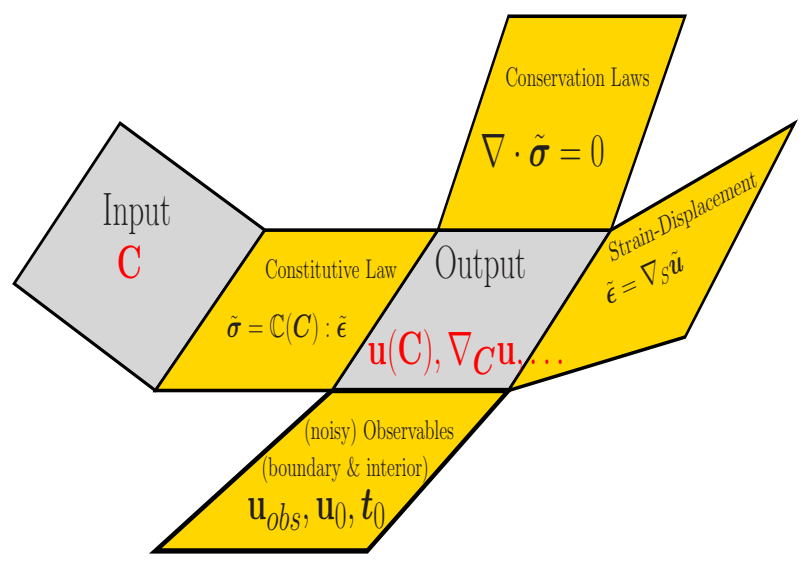

Figure 2: Proposed framework of unfolding the black-box and revealing all model equations.

adopted statistical strategy involves augmenting the relation between data and model predictions with a Gaussian process, which accounts in an additive fashion, for the model error's contribution [49, 57]. However, this explicit additive term may violate physical constraints (e.g. conservation of mass, energy), can get entangled with the measurement error, is not physically interpretable and cumbersome or impractical to infer when it depends on a large number of input parameters [5, 7, 63, 91, 96].

The present paper extends previous work [63] towards developing a novel modeling framework and a set of scalable algorithms that will address the two main challenges in model calibration and validation in PDE-based models, i.e. a) the significant computational cost in problems with an expensive, black-box forward model, and b) the quantification of structural, model uncertainty and its effect on model calibration and predictive estimates. This paper advocates a new paradigm that goes beyond the standard black-box setting (Figure 1) employed thus far. Such a shift is necessitated by the need to quantify model discrepancies in a physically relevant manner. It is based on bringing all model equations in the forefront and, in a Bayesian fashion, quantifying their relative validity using the language of probabilities. As we show in the next sections, this recasts the solution of, even deterministic, forward problems as problems of probabilistic inference. More importantly, in the context of inverse problems, it leads to an augmented posterior that involves all of the state variables of the forward model. Nevertheless, it untangles their complex relations into local terms (Figure 2) and yields a well-posed inverse problem, even in cases when the forward problem is not (e.g. due to incomplete boundary conditions). We demonstrate the potential of this framework in the context of biomechanics where the solution of the aforementioned issues can significantly impact progress in the non-invasive, diagnostic capabilities and assist in the development of patient-specific treatment strategies.

The paper is organized as follows. In section 2, we present the proposed probabilistic framework in the context of linear elastostatics. We introduce an undirected 
probabilistic model that encapsulates all model equations and discuss the recasting of forward and inverse problems as probabilistic inference tasks. In section 3, we present an efficient computational framework for carrying out the aforementioned inference tasks based on Stochastic Variational Inference. The scheme introduced employs a twofold variational approximation which is capable of dealing with the intractable normalization constant in the model-informed prior density. Lastly, the feasibility of the framework is demonstrated by several numerical examples in section 4 . 


\section{Proposed modeling framework}

We begin the elaboration of the proposed model in the context of linear, elliptic PDEs as those arising in small deformation, linear elasticity. Similar models appear for example in heat diffusion or Darcy flow and can be treated similarly even though the physical meaning of the state variables is different. Furthermore, the extension to nonlinear elliptic PDEs as those for example appearing in the context of large deformation, nonlinear elasticity (geometric and constitutive nonlinearities) requires some technical alterations which are not discussed. Further extensions to time-dependent, nonlinear models, which are also of interest (e.g. harmonic and transient elastography), are deferred to a future paper. With regards to the notations adopted we generally adhere to the following rules:

- with boldface, we denote vectors/vector fields.

- with we denote scalar/vector fields.

- with upper-case, we denote random vectors/variables.

- with lower-case, we denote values taken by vectors/variables.

Almost all problems in continuum thermodynamics share a common structure which consists of:

1) conservation law(s) that arise from physical principles and are generally wellfounded and trusted. In the case of biomechanics this amounts to the conservation of linear momentum:

$$
\nabla \cdot \tilde{\boldsymbol{\sigma}}(\boldsymbol{x})=0 \quad \forall \boldsymbol{x} \in \Omega,
$$

where $\tilde{\boldsymbol{\sigma}}(\boldsymbol{x})$ is the Cauchy stress tensor at a point $\boldsymbol{x}$ in the problem domain $\Omega$. Conservation laws serve as the skeleton in many other problems i.e. the conservation of mass/energy in the case of diffusion/advection of mass or heat flow through a (porous) medium. Discretized versions of the aforementioned PDEs are employed, which naturally introduce discretization error.

2) equation(s) of state/constitutive law(s)/closure(s). Constitutive equations refer in general to relations between conjugate thermodynamic variables, such as the stress and strain tensors in bio/solid mechanics. In linear elasticity such relations can be expressed as:

$$
\tilde{\boldsymbol{\sigma}}(\boldsymbol{x})=\mathbb{C}(\boldsymbol{x}): \tilde{\boldsymbol{\epsilon}}(\boldsymbol{x}) \quad \forall \boldsymbol{x} \in \Omega,
$$

where $\mathbb{C}(\boldsymbol{x})$ is the elasticity tensor and $\tilde{\boldsymbol{\epsilon}}(\boldsymbol{x})$ the inifnitesimal strain tensor, which relates to the displacements $\tilde{\boldsymbol{u}}(\boldsymbol{x})$ as follows:

$$
\tilde{\boldsymbol{\epsilon}}(\boldsymbol{x})=\frac{1}{2}\left(\nabla \tilde{\boldsymbol{u}}(\boldsymbol{x})+(\nabla \tilde{\boldsymbol{u}}(\boldsymbol{x}))^{T}\right)=\nabla_{S} \tilde{\boldsymbol{u}}(\boldsymbol{x}) .
$$

Similar constitutive relations have been established between velocity and pressure in flow through permeable media, or flux and temperature in heat diffusion, involving permeability or conductivity tensors. In the context of elastography, our goal 
is to estimate $\mathbb{C}$ and its spatial variability. Constitutive models are largely phenomenological and therefore represent the primary source of model error/uncertainty.

3) observables in the form of boundary/interior conditions or initial conditions in time-dependent problems. In a typical, static forward problem of biomechanics these consist of boundary displacements (Dirichlet boundary conditions) on the part of the boundary $\Gamma_{D B C} \subseteq \partial \Omega$ :

$$
\tilde{\boldsymbol{u}}(\boldsymbol{x})=\boldsymbol{u}_{0}(\boldsymbol{x}) \quad \forall \boldsymbol{x} \in \Gamma_{D B C}
$$

and, potentially also, traction (Neumann) boundary conditions on the part of the boundary $\Gamma_{N B C} \subset \partial \Omega$ :

$$
\tilde{\boldsymbol{\sigma}}(\boldsymbol{x}) \cdot \boldsymbol{n}(\boldsymbol{x})=\boldsymbol{t}_{0}(\boldsymbol{x}) \quad \forall \boldsymbol{x} \in \Gamma_{N B C},
$$

where $\boldsymbol{n}(\boldsymbol{x})$ denotes the unit outward normal vector. These boundary traction$\mathrm{s} /$ displacements might be specified deterministically or stochastically and the wellposedness of the forward problem necessitates that $\Gamma_{D B C} \cup \Gamma_{N B C}=\partial \Omega$ and $\Gamma_{D B C} \cap \Gamma_{N B C}=\emptyset$. In the context of the inverse problems considered these are complemented by observations of interior displacements which we denoted by $\boldsymbol{u}_{\text {obs }}$. These are obtained by employing image processing techniques to the undeformed and deformed (e.g. ultrasound) images of the tissue ([92]). In general, the observables $\boldsymbol{u}_{\text {obs }}$ (and potentially also $\boldsymbol{u}_{0}, \boldsymbol{t}_{0}$ ) are contaminated by noise and represent the primary source of observation error.

Existing deterministic or stochastic (Bayesian) strategies for the solution of the associated inverse problem are based on a premise of a perfect model (up to discretization errors) and a well-posed forward problem i.e. the specification of boundary conditions that ensure the existence/uniqueness of solution. In bio/solid mechanics, a forward solver is usually obtained, upon discretization of the governing equations, in terms of displacements $\tilde{\boldsymbol{u}}(\boldsymbol{x}) \underset{\text { discretize }}{\longrightarrow} \boldsymbol{u}$ and of the constitutive parameters in $\mathbb{C}(\boldsymbol{x}) \underset{\text { discretize }}{\longrightarrow} \boldsymbol{C}$. Traditional Bayesian formulations $[49,57]$ postulate a relation for the observed displacements $\boldsymbol{u}_{\text {obs }}$ of the form:

$$
\boldsymbol{u}_{o b s}=\boldsymbol{u}(\boldsymbol{C})+\boldsymbol{\eta}, \quad \boldsymbol{\eta} \sim \mathcal{N}\left(\mathbf{0}, \sigma_{\eta}^{2} \boldsymbol{I}\right)
$$

where $\boldsymbol{\eta}$ is the observation noise. Solution strategies treat the forward solver that computes the parameter-to-state map $\boldsymbol{u}(\boldsymbol{C})$ as a black-box (Figure 1). Often, gradients $\nabla_{\boldsymbol{C}} \boldsymbol{u}(\boldsymbol{C})$ are available through adjoint formulations [77]. Nevertheless, the number of forward solutions required can become extremely large and scales poorly with the dimension of the unknowns $\boldsymbol{C}$ as discussed in the introduction. In addition to these deficiencies, such a formulation lacks the ability to quantify model errors and while estimates for $\boldsymbol{C}$ can always be obtained, these are obviously invalid if they are based on an incorrect model. The use of an additional term of the form $\boldsymbol{\delta}(\boldsymbol{C})$ or $\boldsymbol{\delta}(\boldsymbol{x})$ in Equation (6) as in [57] may violate physical constraints, can get entangled with the 
measurement error, is not physically interpretable and cumbersome or impracticable when $\boldsymbol{C}$ is high-dimensional as in cases of practical interest [72]. Furthermore, when uncertainty is present with regards to the boundary conditions, these must also be included in the vector of unknowns and be inferred from the data, which introduces additional difficulties.

In contrast, our strategy attempts to break open the black-box model (Figure 2) and bring to the surface all quantities/fields involved in the mathematical description of the physical process of deformation. Under this framework, the solution of both forward and inverse problems is recast as one of statistical inference [19, 32, 47, 48] and we attempt to find all latent, unobserved quantities that are compatible not only with the observables, but with the physics-based-model equations as well. Their reliability, or absence thereof, is expressed in terms of probabilities, allowing one to quantify all error sources such as discretization and structural, model errors.

The formulation advocated consists of three pivotal components which are discussed in the sequel, namely:

1) an augmented prior and posterior distribution dictated by the model equations (section 2.1).

2) the representation (discretization) of the unknown state variables (section 2.2).

3) the solution as probabilistic inference (section 2.3).

\section{$2.1 \quad$ Augmented prior/posterior densities}

In contrast to existing Bayesian formulations which prescribe prior densities merely encapsulating beliefs (in our case, the elastic material properties $\boldsymbol{C}$ ), we advocate a prior model that incorporates the relations and dependencies between system states as implied by the governing equations, i.e. conservation \& constitutive laws. In particular, we view each of those equations as a source of information ${ }^{2}$ and we employ a model interrogation scheme $[19,21]$ in order to extract it. For the conservation of linear momentum (Equation (1)) and without loss of generality, these interrogations can be performed using the Method of Weighted Residuals (MWR, [39]), according to which, for each weighting function $\boldsymbol{w}^{\left(i_{e}\right)}(\boldsymbol{x})$ such that $\boldsymbol{w}^{\left(i_{e}\right)}(\boldsymbol{x})=0$ on $\Gamma_{D B C}$, the weighted residual $r_{e}^{\left(i_{e}\right)}(\tilde{\boldsymbol{\sigma}}(\boldsymbol{x}))$ is (using indicial notation):

$$
\begin{aligned}
r_{e}^{\left(i_{e}\right)}(\tilde{\boldsymbol{\sigma}}(\boldsymbol{x})) & =\int_{\Omega} \boldsymbol{w}_{j}^{\left(i_{e}\right)} \tilde{\boldsymbol{\sigma}}_{j i, i} d V \\
& =\int_{\Gamma_{N B C}} \boldsymbol{w}_{j}^{\left(i_{e}\right)} \tilde{\boldsymbol{\sigma}}_{j i} \boldsymbol{n}_{i} d \Gamma-\int_{\Omega} \boldsymbol{w}_{j, i}^{\left(i_{e}\right)} \tilde{\boldsymbol{\sigma}}_{j i} d V \\
& =\int_{\Gamma_{N B C}} \boldsymbol{w}_{j}^{\left(i_{e}\right)} \boldsymbol{t}_{0, j} d \Gamma-\int_{\Omega} \boldsymbol{w}_{j, i}^{\left(i_{e}\right)} \tilde{\boldsymbol{\sigma}}_{j i} d V \quad \text { (from Equation (5)). }
\end{aligned}
$$

Rather than setting each of these residuals $\left\{r_{e}^{\left(i_{e}\right)}\right\}_{i_{e}=1}^{N_{e}}$ to zero, we use them to induce a probability measure on candidate stress fields $\tilde{\boldsymbol{\sigma}}(\boldsymbol{x})$. Such measures should

\footnotetext{
${ }^{2}$ It is due to this different perspective in the interpretation of solutions of PDEs that we refrain from the classical mathematical treatment of trial and weighting function spaces.
} 


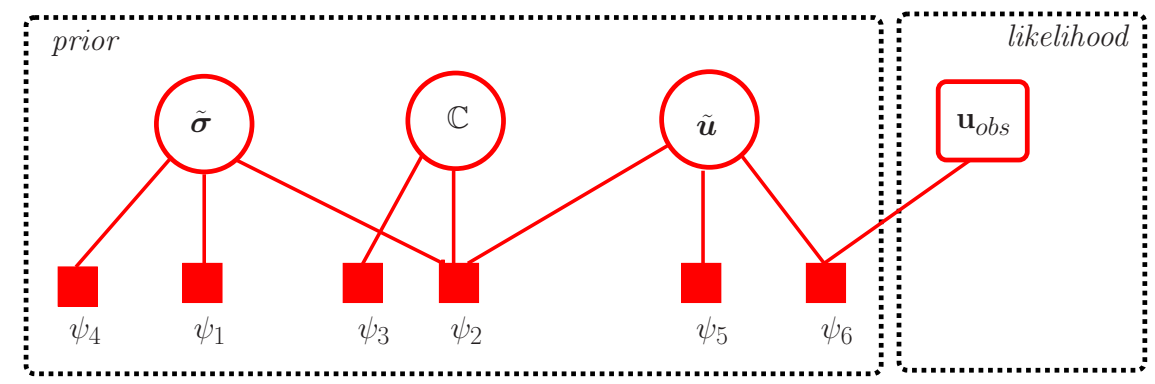

Figure 3: Factor graph exhibiting the augmented state space in the case of linear elasticity. Round nodes represent variables, square nodes represent factors. There is an edge from each variable to every factor that mentions it. The factor potentials $\psi_{i}$ arise from model equations (conservation \& constitutive laws) as well as observations.

contract to a Dirac measure around the true, but unknown, stress field, as $N_{e} \rightarrow \infty$. Our strategy resembles conceptually the emerging field known as Probabilistic Numerics $[22,47]$. In particular, if $\epsilon$ is analogous to the numerical tolerance employed in deterministic schemes to enforce zero residuals, we can define a Gaussian probability density $\mathcal{N}\left(0, \epsilon^{2}\right)$ for $r_{e}^{\left(i_{e}\right)}$ which in turn implies a probability measure on the space of candidate solutions $\tilde{\boldsymbol{\sigma}}(\boldsymbol{x})$. The combination of $N_{e}$ such relations yields a density for $\tilde{\boldsymbol{\sigma}}(\boldsymbol{x})$ of the form ${ }^{3}$ (we omit dependence on $\epsilon$ for simplicity):

$$
\begin{array}{rlrl}
p_{e}(\tilde{\boldsymbol{\sigma}}(\boldsymbol{x})) & \propto \psi_{1}(\tilde{\boldsymbol{\sigma}}(\boldsymbol{x})) & \text { (see Figure 3) } \\
& =\prod_{i_{e}=1}^{N_{e}} \mathcal{N}\left(r_{e}^{\left(i_{e}\right)}(\tilde{\boldsymbol{\sigma}}(\boldsymbol{x})) ; 0, \epsilon^{2}\right) .
\end{array}
$$

This scores candidate stress fields $\tilde{\boldsymbol{\sigma}}(\boldsymbol{x})$ according to their satisfaction of the governing equation (in the MWR form). Fields that yield 0 residuals get the highest score and, the more weighting functions $N_{e}$ are used, the narrower becomes the region in the space of trial solutions where the probability mass is concentrated. We note at this stage that the MWR considered is not at all restrictive as (at least) six methods (i.e. collocation, sub-domain, least-squares, (Petrov)-Galerkin, moments) can be considered as special cases by appropriate selection of the $\boldsymbol{w}^{(i)}(\boldsymbol{x})$.

Enforcing any reliable model equation (e.g. strain-displacement relation, incompressibility) can also be done in a similar manner by artificially defining a probability measure as above which can degenerate to a Dirac measure as the precision parameter $\epsilon$ decays. This is particularly useful when it comes to the second piece in the puzzle which arises from the constitutive equations and requires the specification of the strain tensor $\tilde{\boldsymbol{\epsilon}}(\boldsymbol{x})$. This can be indirectly expressed by a probabilistic enforcement of the strain-displacements relation in Equation (3) or directly in terms of the displacement field $\tilde{\boldsymbol{u}}(\boldsymbol{x})$. If one enforces Equation (2) point-wise at $\left\{\boldsymbol{x}^{\left(i_{c}\right)}\right\}_{i_{c}=1}^{N_{c}}$, then at each of those points, the residual $\boldsymbol{r}_{c}^{\left(i_{c}\right)}$ :

$$
\boldsymbol{r}_{c}^{\left(i_{c}\right)}(\tilde{\boldsymbol{\sigma}}(\boldsymbol{x}), \tilde{\boldsymbol{u}}(\boldsymbol{x}), \mathbb{C}(\boldsymbol{x}))=\tilde{\boldsymbol{\sigma}}\left(\boldsymbol{x}^{\left(i_{c}\right)}\right)-\mathbb{C}\left(\boldsymbol{x}^{\left(i_{c}\right)}\right): \nabla_{S} \tilde{\boldsymbol{u}}\left(\boldsymbol{x}^{\left(i_{c}\right)}\right),
$$

\footnotetext{
${ }^{3}$ In the following, we abuse notation by defining probability densities on functions/fields in order to simplify the presentation. In computational implementations, these fields are discretized as discussed in section 3 .
} 
which quantifies the discrepancy between the actual stresses and the constitutive model predicted stresses, is zero only in the case of a perfect model. As the validity of the model is unknown a priori, we propose a hierarchical prior of the form:

$$
p\left(\boldsymbol{r}_{c}^{\left(i_{c}\right)} \mid \Lambda_{i_{c}}\right)=\mathcal{N}\left(\mathbf{0}, \Lambda_{i_{c}}^{-1} \boldsymbol{I}\right) .
$$

The simplest such model utilizes a single hyper-parameter $\Lambda_{i_{c}}$ per point $\boldsymbol{x}^{\left(i_{c}\right)}$, which reflects the magnitude of the constitutive model error. Multivariate representations as well as more complex model-error distributions are also possible. Large values of $\Lambda_{i_{c}}^{-1}$ indicate locations where the model error is high and vice versa. We emphasize that nonzero values of $\boldsymbol{r}_{c}^{\left(i_{c}\right)}$ do not arise due to variability in the material parameters $\mathbb{C}(\boldsymbol{x})$, but rather due to the inadequacy of the constitutive model to provide sufficient closures to the governing equations. As discussed earlier, Equation (10) employed over all enforcement points $N_{c}$ implies a (joint) probability density over the fields $\tilde{\boldsymbol{\sigma}}(\boldsymbol{x}), \tilde{\boldsymbol{u}}(\boldsymbol{x}), \mathbb{C}(\boldsymbol{x})$ (see Figure 3$)$ :

$$
\begin{aligned}
p_{c}(\tilde{\boldsymbol{\sigma}}(\boldsymbol{x}), \tilde{\boldsymbol{u}}(\boldsymbol{x}), \mathbb{C}(\boldsymbol{x}) \mid \boldsymbol{\Lambda}) & \propto \psi_{2}(\tilde{\boldsymbol{\sigma}}(\boldsymbol{x}), \tilde{\boldsymbol{u}}(\boldsymbol{x}), \mathbb{C}(\boldsymbol{x})), \quad \boldsymbol{\Lambda}=\left\{\Lambda_{i_{c}}\right\}_{i_{c}=1}^{N_{c}} \\
& =\prod_{i_{c}=1}^{N_{c}} \mathcal{N}\left(\boldsymbol{r}_{c}^{\left(i_{c}\right)}(\tilde{\boldsymbol{\sigma}}(\boldsymbol{x}), \tilde{\boldsymbol{u}}(\boldsymbol{x}), \mathbb{C}(\boldsymbol{x})) ; \quad \mathbf{0}, \Lambda_{i_{c}}^{-1} \boldsymbol{I}\right) .
\end{aligned}
$$

The aforementioned densities can be straightforwardly complemented by "traditional" priors employed in canonical Bayesian formulations [4], e.g.:

$$
p_{C}\left(\mathbb{C}(\boldsymbol{x}) \mid \boldsymbol{\theta}_{C}\right) \propto \psi_{3}(\mathbb{C}(\boldsymbol{x})) \quad(\text { see Figure 3) }
$$

for the unknown material property field $\mathbb{C}(\boldsymbol{x})$ with hyperparameters $\boldsymbol{\theta}_{C}$. Similarly, if other prior information is available about the other state variables $\tilde{\boldsymbol{\sigma}}(\boldsymbol{x}), \tilde{\boldsymbol{u}}(\boldsymbol{x})$, these can be incorporated e.g. as:

$$
p_{\sigma}\left(\tilde{\boldsymbol{\sigma}}(\boldsymbol{x}) \mid \boldsymbol{\theta}_{\sigma}\right) \propto \psi_{4}(\tilde{\boldsymbol{\sigma}}(\boldsymbol{x}))
$$

and:

$$
p_{u}\left(\tilde{\boldsymbol{u}}(\boldsymbol{x}) \mid \boldsymbol{\theta}_{u}\right) \propto \psi_{5}(\tilde{\boldsymbol{u}}(\boldsymbol{x})) .
$$

The combination of Equations (8), (11) as well as (11), (13), (14), define a joint prior for the state variables of the following form (we omit hyperparameters $\boldsymbol{\theta}_{C}, \boldsymbol{\theta}_{\sigma}, \boldsymbol{\theta}_{u}$ for simplicity):

$$
\begin{aligned}
p(\tilde{\boldsymbol{\sigma}}(\boldsymbol{x}), \tilde{\boldsymbol{u}}(\boldsymbol{x}), \mathbb{C}(\boldsymbol{x}) \mid \boldsymbol{\Lambda}) & =\frac{1}{Z(\boldsymbol{\Lambda})} \prod_{k=1}^{5} \psi_{k}(\tilde{\boldsymbol{\sigma}}(\boldsymbol{x}), \tilde{\boldsymbol{u}}(\boldsymbol{x}), \mathbb{C}(\boldsymbol{x})) \\
& =\frac{\pi_{\boldsymbol{\Lambda}}(\tilde{\boldsymbol{\sigma}}(\boldsymbol{x}), \tilde{\boldsymbol{u}}(\boldsymbol{x}), \mathbb{C}(\boldsymbol{x}))}{Z(\boldsymbol{\Lambda})} \\
& =\frac{1}{Z(\boldsymbol{\Lambda})} p_{e}(\tilde{\boldsymbol{\sigma}}(\boldsymbol{x})) \quad p_{c}(\tilde{\boldsymbol{\sigma}}(\boldsymbol{x}), \tilde{\boldsymbol{u}}(\boldsymbol{x}), \mathbb{C}(\boldsymbol{x})) \\
& \times p_{C}\left(\mathbb{C}(\boldsymbol{x}) \mid \boldsymbol{\theta}_{C}\right) p_{\sigma}\left(\tilde{\boldsymbol{\sigma}}(\boldsymbol{x}) \mid \boldsymbol{\theta}_{\sigma}\right) p_{u}\left(\tilde{\boldsymbol{u}}(\boldsymbol{x}) \mid \boldsymbol{\theta}_{u}\right),
\end{aligned}
$$

where $Z(\boldsymbol{\Lambda})$ is the normalization constant ${ }^{4}$.

\footnotetext{
${ }^{4}$ In the absence of specific information, very vague Gaussians can be used for the priors in Equations (11), (13), (14) to ensure integrability, i.e. $Z(\boldsymbol{\Lambda})<+\infty$
} 
We note that the prior model implies an undirected probabilistic graphical model [61] which takes the form of a factor graph between state variables (Figure 3). We emphasize that this does not rely on the availability of a well-posed forward model (e.g. one would notice the absence of boundary conditions since these are data and will be treated in the likelihood), but rather invokes (in a probabilistic fashion) relationships between the system's states as suggested by the model's equations. More importantly, it relieves the formulation of the black-box, input-output relation $\boldsymbol{u}(\boldsymbol{C})$ as in Equation (6). Dependencies between all state variables appear explicitly in the factors making up Equation (15). As explained in section 2.3, this will allow significant accelerations in the inference task, despite the augmented state space in comparison to standard deterministic or Bayesian formulations. We should also note that the prior model effectively depends on the number of interrogations $N_{e}$ and $N_{c}$ of the governing equations. The larger $N_{e}, N_{c}$ become, the more tightly should the prior concentrate around the continuous, model-dictated values. In contrast, the smaller $N_{e}, N_{c}$ are, the flatter the prior becomes as the number of equations enforced is smaller. This provides an added advantage in the inference task (section 2.3), as these numbers define effectively a tempering schedule, from simpler to more complex densities [62].

The incorporation of various error sources in the prior model enables one to properly account for the observation error in the data. Given displacement data (interior or boundary) $\boldsymbol{u}_{\text {obs }}=\left\{\boldsymbol{u}_{o b s, i_{o}}\right\}_{i_{o}=1}^{N_{o}}$ at $N_{o}$ locations $\boldsymbol{x}^{\left(i_{o}\right)}$, then:

$$
\boldsymbol{u}_{o b s, i_{o}}=\tilde{\boldsymbol{u}}\left(\boldsymbol{x}^{\left(i_{o}\right)}\right)+\eta_{i_{o}}, \quad \eta_{i_{o}} \sim \mathcal{N}\left(0, \sigma_{\eta}^{2}\right), \quad i_{0}=1, \ldots, N_{o},
$$

where $\sigma_{\eta}^{2}$ is the variance of the observation noise. We emphasize here a fundamental difference with the analogous Equation (6) used in traditional Bayesian formulations. Therein, the $\eta$ 's express the difference between observables and displacements predicted by the model which is assumed to be perfect, whereas here the model-related equations have been sequestered in the prior (Equation (15)). The Gaussian assumption for the $\eta_{i_{o}}$ is not restrictive as in Equation (6), where it must simultaneously account for model errors. The equation above yields a likelihood:

$$
\begin{aligned}
p\left(\boldsymbol{u}_{o b s} \mid \tilde{\boldsymbol{u}}(\boldsymbol{x})\right) & \propto \psi_{6}(\tilde{\boldsymbol{u}}(\boldsymbol{x})) \\
& =\prod_{i_{o}=1}^{N_{o}} \mathcal{N}\left(\boldsymbol{u}_{o b s, i_{o}} ; \tilde{\boldsymbol{u}}\left(\boldsymbol{x}^{\left(i_{o}\right)}\right), \sigma_{\eta}^{2}\right),
\end{aligned}
$$

which, as is suggested in Figure 3, gives rise to an augmented posterior density over all state variables as follows:

$$
\begin{aligned}
p\left(\tilde{\boldsymbol{\sigma}}(\boldsymbol{x}), \tilde{\boldsymbol{u}}(\boldsymbol{x}), \mathbb{C}(\boldsymbol{x}) \mid \boldsymbol{u}_{o b s}, \boldsymbol{\Lambda}\right) & \propto \prod_{k=1}^{6} \psi_{k}(\tilde{\boldsymbol{\sigma}}(\boldsymbol{x}), \tilde{\boldsymbol{u}}(\boldsymbol{x}), \mathbb{C}(\boldsymbol{x})) \\
& \propto \underbrace{p\left(\boldsymbol{u}_{\text {obs }} \mid \tilde{\boldsymbol{u}}(\boldsymbol{x})\right)}_{\text {likelihood Equation (17) }} \underbrace{p(\tilde{\boldsymbol{\sigma}}(\boldsymbol{x}), \tilde{\boldsymbol{u}}(\boldsymbol{x}), \mathbb{C}(\boldsymbol{x}) \mid \boldsymbol{\Lambda})}_{\text {prior Equation (15) }}
\end{aligned}
$$

Despite the superficial complexity of the posterior density above, we note that this consists of large products, of tractable, local terms nevertheless. The absence of any "black-box" terms is the key to facilitating the inference process. 


\subsection{Representation of the unknown state variables}

The presentation of the augmented prior/posterior densities in the previous section was done (with some abuse of notation) in infinite dimensions, i.e. without presupposing a particular discretization of the latent state fields. A possibility for carrying out inference tasks is the utilization of Gaussian Processes that are frequently employed for nonparametric representations (e.g. [19]). We believe though that in the current setting such a choice would be impracticable due to the non-Gaussian terms in Equation (18). In order to carry computations, we advocate discretized representations which will unavoidably introduce discretization errors. We note here that such a representation of the latent fields $\tilde{\boldsymbol{\sigma}}(\boldsymbol{x}), \tilde{\boldsymbol{u}}(\boldsymbol{x}), \mathbb{C}(\boldsymbol{x})$, is not dependent on the discretization of the governing equations, which is controlled by the number of weighting functions $N_{e}$ (Equation (8)) and the number of (effectively collocation) points $N_{c}$, where the constitutive equation is interrogated (Equation (11)). This decoupling is an additional advantage of the proposed strategy that can lead to adaptive, multiresolution inference as discussed in the conclusions.

In the framework proposed, we seek all problem fields, i.e. the stresses $\tilde{\boldsymbol{\sigma}}(\boldsymbol{x})$, displacements $\tilde{\boldsymbol{u}}(\boldsymbol{x})$, and constitutive model parameters $\mathbb{C}(\boldsymbol{x})$ and employ a (potentially overcomplete) dictionary of feature functions $\boldsymbol{f}(\boldsymbol{x})=\left\{f_{u, j}(\boldsymbol{x}), f_{C, j}(\boldsymbol{x}), f_{\sigma, j}(\boldsymbol{x})\right\}$ such that:

$$
\begin{aligned}
& \tilde{\boldsymbol{u}}(\boldsymbol{x})=\sum_{j=1}^{M_{u}} \boldsymbol{U}_{j} f_{u, j}(\boldsymbol{x}), \\
& \tilde{\mathbb{C}}(\boldsymbol{x})=\sum_{j=1}^{M_{C}} \mathbb{C}_{j} f_{C, j}(\boldsymbol{x}), \\
& \tilde{\boldsymbol{\sigma}}(\boldsymbol{x})=\sum_{j=1}^{M_{\sigma}} \boldsymbol{\Sigma}_{j} f_{\sigma, j}(\boldsymbol{x}),
\end{aligned}
$$

where, in general, $\boldsymbol{U}_{j}$ are vector-valued, $\boldsymbol{\Sigma}_{j}$ second-order-tensor-valued and $\mathbb{C}_{j}$ fourthorder-tensor-valued coefficients. For a fixed set of functions $\boldsymbol{f}(\boldsymbol{x})$ this would imply an augmented posterior on the state vector $\{\boldsymbol{U}, \boldsymbol{C}, \boldsymbol{\Sigma}\}$, where $\boldsymbol{U}=\left\{\boldsymbol{U}_{j}\right\}_{j=1}^{M_{u}}, \boldsymbol{C}=$ $\left\{\mathbb{C}_{j}\right\}_{j=1}^{M_{C}}, \boldsymbol{\Sigma}=\left\{\boldsymbol{\sigma}_{j}\right\}_{j=1}^{M_{\sigma}}$ arising from the substitution of Equation (19) into the respective equations, e.g. Equation (15) or Equation (18).

On one hand, it is desirable to maximize the expressivity of such representations in order to minimize the discretization error. On the other, by increasing the dimensionality of the unknown parameters in the representation, not only one impedes computations, but can potentially lead to a multi-modal posterior, especially when the number of unknowns is much larger than the data $N_{o}$ and $N_{e}+N_{c}$.

This poses an interesting model selection problem. One option to address this is to initiate the search with a small number of feature functions and progressively add more. These can be selected from a pool of candidates by employing appropriate criteria $[9,30]$. Another alternative is to preselect a large, overcomplete set of feature functions and prune them by enforcing sparsity. In the Bayesian setting advocated, this can be readily achieved by employing sparsity-promoting hierarchical priors (e.g. spike-and-slab [54], automatic relevance determination [67, 102], horseshoe [18]). The underlying assumption is that the fields of interest are highly compressible, i.e. most of the coefficients $\boldsymbol{U}_{j}, \mathbb{C}_{j}, \boldsymbol{\Sigma}_{j}$ are zero [17, 34,66]. Sparsity as a principle of parsimony has successfully been applied in Bayesian formulations in various contexts such 
the representation of natural images [80] or the solution of PDE-based inverse problems [41] and a host of algorithms have been developed, not only for finding such representations, but also an appropriate dictionary for achieving this goal [66].

In this work, we adopt a simpler strategy that employs feature functions from traditional finite element analysis (FEA). In particular we employ irregular triangulations $\mathcal{T}=\cup_{e}^{n_{e l}} \Omega_{e}$ (in 2D) of the problem domain and feature functions $f_{C, j}(\boldsymbol{x}), f_{\sigma, j}(\boldsymbol{x})$ that are constant within each triangle $\Omega_{e}$ i.e.:

$$
f_{C, j}(\boldsymbol{x})=\left\{\begin{array}{ll}
1, & \text { if } \boldsymbol{x} \in \Omega_{j} \\
0, & \text { otherwise, }
\end{array} \quad f_{\sigma, j}(\boldsymbol{x})= \begin{cases}1, & \text { if } \boldsymbol{x} \in \Omega_{j} \\
0, & \text { otherwise }\end{cases}\right.
$$

As for the feature function $f_{u, j}$, we employ the piece-wise linear shape functions corresponding to linear triangular elements from finite element analysis [53]. A direct implication of this choice is that the coefficients $\boldsymbol{U}_{j}$ correspond to the displacements at the vertices of each triangle (also called nodal displacements). Furthermore, the corresponding strain tensor $\tilde{\boldsymbol{\epsilon}}$ computed from such a displacement field though Equation (3) would also be piece-wise constant (as $\tilde{\boldsymbol{\sigma}}$ and $\tilde{\mathbb{C}}$ ). As a result, enforcement of the constitutive law as in Equation (11), can be effectively done on an element-byelement basis (i.e. the number of such contributions $N_{c}$ is at most equal to the number of elements/triangles $n_{e l}$ ). We do not claim that such a representation is optimal or the sparsest possible and that it unavoidably introduces a discretization error. We note though that given a sufficiently fine triangulation, one can approximate arbitrary close the true stess, displacement and material parameter fields, which are generally not piece-wise constant, nor piece-wise linear. We assume that the discretization in the problems considered are sufficiently fine to ignore the discretization error which would be smaller than the other contributions.

The weighting functions $\boldsymbol{w}(\boldsymbol{x})$ employed in the MWR as in Equation (7) are also given by the piece-wise linear shape functions of linear triangular elements (as for $f_{u, j}$ ). Hence, each residual in Equation (7) corresponds to enforcing equilibrium of forces at each vertex/node. The locality of feature and weighting functions endows the formulation with the computational conveniences of FEA, particularly with the computation of residuals which can also be done on an element basis and make use of existing computer codes. In particular, we note that based on the aforementioned discussion:

- we denote with $\boldsymbol{U} \in \mathbb{R}^{n_{u}}$ the vector for the discretized representation of the displacement field $\tilde{\boldsymbol{u}}(\boldsymbol{x})$. It consists of the displacements at each of the $n_{V}$ vertices/nodes, i.e. $\boldsymbol{U}=\left\{\boldsymbol{U}_{j}\right\}_{j=1}^{n_{V}}$. The strains obtained from Equation (3) will be piece-wise constant. We denote by $\boldsymbol{E}_{e}$ the strains corresponding to each element $e$. These can be readily computed from the nodal displacements of the element, say $\boldsymbol{U}_{e}$, by employing the well-known in FEA strain-displacement matrix $\boldsymbol{B}_{e}$ as $\boldsymbol{E}_{e}=\boldsymbol{B}_{e} \boldsymbol{U}_{e}$.

- we denote with $C \in \mathbb{R}^{n_{C}}$ the vector for the discretized representation of the elastic tensor field $\mathbb{C}(\boldsymbol{x})$. It can also be partitioned on an element/triangle basis as $\boldsymbol{C}=\left\{\boldsymbol{C}_{e}\right\}_{e=1}^{n_{e l}}$. 
- we denote with $\Sigma \in \mathbb{R}^{n_{\sigma}}$ the vector for the discretized representation of the stress field $\tilde{\boldsymbol{\sigma}}(\boldsymbol{x})$. Given the piece-wise constant nature of this representation, it can also be partitioned on an element/triangle basis as $\boldsymbol{\Sigma}=\left\{\boldsymbol{\Sigma}_{e}\right\}_{e=1}^{n_{e l}}$.

- the enforcement of the conservation of linear momentum as in Equation (7) for each weighting function $\boldsymbol{w}^{\left(i_{e}\right)}(\boldsymbol{x})$ can be expressed as:

$$
r_{e}^{\left(i_{e}\right)}(\tilde{\boldsymbol{\sigma}}(\boldsymbol{x}))=f_{i_{e}}-\boldsymbol{b}_{i_{e}}^{T} \boldsymbol{\Sigma},
$$

where $f_{i_{e}}=\int_{\Gamma_{N B C}} \boldsymbol{w}_{j}^{\left(i_{e}\right)} \boldsymbol{t}_{0, j} d \Gamma$ and $\boldsymbol{b}_{i_{e}}^{T} \boldsymbol{\Sigma}=\int_{\Omega} \boldsymbol{w}_{j, i}^{\left(i_{e}\right)} \tilde{\boldsymbol{\sigma}}_{j i} d V$. The vector $\boldsymbol{b}_{i_{e}}$ is constant due to the discretization choices made and can be pre-computed along with the scalar $f_{i_{e}}$. The assembly of all such residuals for $i_{e}=1, \ldots, N_{e}$ as in Equation (8) gives rises to the following factor potential $\psi_{1}$ :

$$
\psi_{1}(\boldsymbol{\Sigma})=e^{-\frac{1}{2 \epsilon^{2}}\left\|\boldsymbol{f}-\boldsymbol{B}^{T} \boldsymbol{\Sigma}\right\|^{2}} .
$$

- the enforcement of the constitutive law as in Equation (9) over each triangle/element $e$ can be expressed as:

$$
\boldsymbol{r}_{c}^{(e)}(\tilde{\boldsymbol{\sigma}}(\boldsymbol{x}), \tilde{\boldsymbol{u}}(\boldsymbol{x}), \mathbb{C}(\boldsymbol{x}))=\boldsymbol{\Sigma}_{e}-\boldsymbol{C}_{e} \boldsymbol{B}_{e} \boldsymbol{U}_{e} .
$$

The assembly of all such residuals for $e=1, \ldots, n_{e l}$ as in Equation (11) gives rise to the following factor potential $\psi_{2}$ :

$$
\psi_{2}(\boldsymbol{\Sigma}, \boldsymbol{C}, \boldsymbol{U} ; \boldsymbol{\Lambda})=\prod_{e=1}^{n_{e l}} e^{-\frac{\Lambda_{e}}{2}\left\|\boldsymbol{\Sigma}_{e}-C_{e} B_{e} \boldsymbol{U}_{e}\right\|^{2}} .
$$

- the priors on the material parameters will be specialized in the applications. We denote by $\psi_{3}(\boldsymbol{C})$ the corresponding factor potential as in Equation (12).

- we employ vague Gaussian priors on $\boldsymbol{\Sigma}$ and $\boldsymbol{U}$ as in Equation (13) and Equation (14) respectively. Their role is not to reflect any prior information (which is unavoidably problem dependent), but rather to ensure the integrability of the corresponding augmented prior in Equation (15). The corresponding factor potentials are:

$$
\begin{aligned}
& \psi_{4}(\boldsymbol{\Sigma})=e^{-\frac{\theta_{\sigma}}{2}\|\boldsymbol{\Sigma}\|^{2}}, \\
& \psi_{5}(\boldsymbol{U})=e^{-\frac{\theta_{u}}{2}\|\boldsymbol{U}\|^{2}},
\end{aligned}
$$

where $\theta_{\sigma}, \theta_{u} \rightarrow 0$.

Adaptations to one or three dimensions are straightforward where one can employ the machinery of linear or trilinear hexahedral elements respectively.

\subsection{Rephrasing forward/inverse problems as probabilistic inference tasks}

In this section, we recapitulate the framework introduced in section 2.1 in view of the discretizations adopted in section 2.2, and, more importantly, demonstrate how 
forward and inverse problems can be solved as probabilistic inference tasks, without ever resorting to the black-box PDE solver.

Consider first a typical forward problem where one is given:

- the conservation law of Equation (1), which is enforced as in Equation (7) with $\epsilon \rightarrow 0$ (in practice, very small values are prescribed).

- the constitutive law of Equation (2), which is enforced as in Equation (9) and for the purpose of solving the forward problem is assumed to be valid (i.e. $\left.\Lambda_{i_{c}}=\lambda_{i_{c}} \rightarrow \infty\right)$. In addition, the elasticity tensor $\mathbb{C}(\boldsymbol{x})$ is prescribed, i.e. in the discretized representation $\boldsymbol{C}=\boldsymbol{c}$ where $\boldsymbol{c}$ is known.

- boundary conditions as in Equation (4) or Equation (5). For the sake of simplicity, suppose only Dirichlet boundary conditions are prescribed (i.e. $\boldsymbol{u}_{0}(\boldsymbol{x})$ in Equation (4)), which in the discretized represenation of the displacement field implies that part of the vector $\boldsymbol{U}$ is known. If $\boldsymbol{U}=\left(\boldsymbol{U}_{i}, \boldsymbol{U}_{b}\right)$ is partitioned to interior $\boldsymbol{U}_{i}$ and boundary $\boldsymbol{U}_{b}$ displacements, then $\boldsymbol{U}_{b}=\boldsymbol{u}_{0}$ where $\boldsymbol{u}_{0}$ is known.

The augmented prior presented in Equation (15) and adapted in section 2.2 can be expressed as:

$$
\begin{aligned}
p\left(\boldsymbol{\Sigma}, \boldsymbol{C}, \boldsymbol{U}_{i}, \boldsymbol{U}_{b} \mid \boldsymbol{\Lambda}\right) & =\frac{1}{Z(\boldsymbol{\Lambda})} \psi_{1}(\boldsymbol{\Sigma}) \psi_{2}\left(\boldsymbol{\Sigma}, \boldsymbol{C}, \boldsymbol{U}_{i}, \boldsymbol{U}_{b} ; \boldsymbol{\Lambda}\right) \psi_{3}(\boldsymbol{C}) \psi_{4}(\boldsymbol{\Sigma}) \psi_{5}\left(\boldsymbol{U}_{i}, \boldsymbol{U}_{b}\right) \\
& =\frac{\pi_{\boldsymbol{\Lambda}}\left(\boldsymbol{\Sigma}, \boldsymbol{C}, \boldsymbol{U}_{i}, \boldsymbol{U}_{b}\right)}{Z(\boldsymbol{\Lambda})},
\end{aligned}
$$

where:

$$
\pi_{\boldsymbol{\Lambda}}\left(\boldsymbol{\Sigma}, \boldsymbol{C}, \boldsymbol{U}_{i}, \boldsymbol{U}_{b}\right)=\psi_{1}(\boldsymbol{\Sigma}) \psi_{2}\left(\boldsymbol{\Sigma}, \boldsymbol{C}, \boldsymbol{U}_{i}, \boldsymbol{U}_{b} ; \boldsymbol{\Lambda}\right) \psi_{3}(\boldsymbol{C}) \psi_{4}(\boldsymbol{\Sigma}) \psi_{5}\left(\boldsymbol{U}_{i}, \boldsymbol{U}_{b}\right)
$$

and:

$$
Z(\boldsymbol{\lambda})=\int \psi_{1}(\boldsymbol{\sigma}) \psi_{2}\left(\boldsymbol{\sigma}, \boldsymbol{c}, \boldsymbol{u}_{i}, \boldsymbol{u}_{b} ; \boldsymbol{\lambda}\right) \psi_{3}(\boldsymbol{c}) \psi_{4}(\boldsymbol{\sigma}) \psi_{5}\left(\boldsymbol{u}_{i}, \boldsymbol{u}_{b}\right) d \boldsymbol{\sigma} d \boldsymbol{c} d \boldsymbol{u}_{i} d \boldsymbol{u}_{b}
$$

Under the specifications of the forward problem (i.e. $\boldsymbol{C}=\boldsymbol{c}, \boldsymbol{U}_{b}=\boldsymbol{u}_{0}$ and given $\boldsymbol{\Lambda}=\boldsymbol{\lambda}$ ), the goal is to write the conditional density of the undetermined latent random variables $\boldsymbol{\Sigma}, \boldsymbol{U}_{i}$, i.e.:

$$
\begin{aligned}
p\left(\boldsymbol{\Sigma}, \boldsymbol{U}_{i} \mid \boldsymbol{C}=\boldsymbol{c}, \boldsymbol{U}_{b}=\boldsymbol{u}_{0}, \boldsymbol{\lambda}\right) & \propto \frac{p\left(\boldsymbol{\Sigma}, \boldsymbol{C}=\boldsymbol{c}, \boldsymbol{U}_{i}, \boldsymbol{U}_{b}=\boldsymbol{u}_{0} \mid \boldsymbol{\lambda}\right)}{p\left(\boldsymbol{C}=\boldsymbol{c}, \boldsymbol{U}_{b}=\boldsymbol{u}_{0} \mid \boldsymbol{\lambda}\right)} \\
& =\frac{p\left(\boldsymbol{\Sigma}, \boldsymbol{C}=\boldsymbol{c}, \boldsymbol{U}_{i}, \boldsymbol{U}_{b}=\boldsymbol{u}_{0} \mid \boldsymbol{\lambda}\right)}{\int p\left(\boldsymbol{\sigma}, \boldsymbol{C}=\boldsymbol{c}, \boldsymbol{u}_{i}, \boldsymbol{U}_{b}=\boldsymbol{u}_{0} \mid \boldsymbol{\lambda}\right) d \boldsymbol{\sigma} d \boldsymbol{u}_{i}} \\
& =\frac{\pi_{\boldsymbol{\lambda}}\left(\boldsymbol{\Sigma}, \boldsymbol{C}=\boldsymbol{c}, \boldsymbol{U}_{i}, \boldsymbol{U}_{b}=\boldsymbol{u}_{0}\right)}{\int \pi_{\boldsymbol{\lambda}}\left(\boldsymbol{\sigma}, \boldsymbol{C}=\boldsymbol{c}, \boldsymbol{u}_{i}, \boldsymbol{U}_{b}=\boldsymbol{u}_{0}\right) d \boldsymbol{\sigma} d \boldsymbol{u}_{i}} .
\end{aligned}
$$

Hence, identifying the latent variables $\boldsymbol{\Sigma}, \boldsymbol{U}_{i}$ (i.e. stresses and interior displacements) is equivalent to finding the conditional augmented prior above. This, for example, can 
be performed by sampling (e.g. using MCMC or SMC), which, as can be seen from the form of the factor of potentials, involves explicit terms, i.e. no black-boxes and no calls to a PDE solver. Similarly, derivatives (first and second order) with respect to $\boldsymbol{\Sigma}, \boldsymbol{U}_{i}$ can be readily computed and used to facilitate the sampling/inference process. In the following section, we demonstrate the use of Stochastic Variational Inference tools, which can be used for that purpose and efficiently provide approximations of the sought conditional density.

Other types of boundary conditions can be readily treated in the same fashion. Furthermore, uncertainty propagation tasks can also be dealt efficiently. If, for example, random material properties $\boldsymbol{C}$ were assumed and a density was prescribed, then one could readily sample values, say $\boldsymbol{c}$, for $\boldsymbol{C}$ from this density and subsequently identify the conditional as in Equation (29) for each of these $\boldsymbol{c}^{5}$. We finally note the possibility of solving ill-posed forward problems, e.g. problems where boundary conditions are under- or over-specified. This is because the corresponding augmented density is still well-defined and therefore inference can be readily performed.

Consider now a typical inverse problem where one is given:

- the conservation law of Equation (1), which is enforced as in Equation (7) with $\epsilon \rightarrow 0$ (in practice, very small values are prescribed).

- the constitutive law of Equation (2), which is enforced as in Equation (9), but the validity of which is unknown (i.e. $\Lambda_{i_{c}}$ are unspecified). In addition, the constitutive parameters $\boldsymbol{C}$ are unspecified, in which case $p_{C}\left(\boldsymbol{C} \mid \boldsymbol{\theta}_{C}\right)$ in Equation (12) plays the role of the prior in canonical Bayesian formulations.

- boundary conditions as in Equation (4) or Equation (5). For the sake of simplicity suppose only Dirichlet boundary conditions are prescribed (i.e. $\boldsymbol{u}_{0}(\boldsymbol{x})$ in Equation (4)), which in the discretized represenation of the displacement field implies that $\boldsymbol{U}_{b}=\boldsymbol{u}_{0}$.

- noisy interior displacements $\boldsymbol{u}_{o b s}$ as in Equation (16), which give rise to a likelihood $p\left(\boldsymbol{u}_{o b s} \mid \boldsymbol{U}_{i}\right)$ as in Equation (17) (upon discretization).

In this scenario, the unknown state variables consist of $\boldsymbol{\Sigma}, \boldsymbol{U}_{i}$ and $\boldsymbol{C}$. In addition one must also identify the constitutive model error hyper-parameters $\boldsymbol{\Lambda}$, which are effectively the connecting threads in the probabilistic web of relationships constructed - the same way constitutive laws are necessary to provide closure to the deterministic, PDE-based model. Rather than a hard enforcement though, they control the validity of the constitutive law at each interrogation point $\boldsymbol{x}^{i_{c}}$ and must be learned, simultaneously with the other latent state variables. Clearly, such a problem is underdetermined unless stress and strain data were simultaneously available. Critical to learning meaningful values for these hyperparameters is therefore the selection of an appropriate prior model. A vague such model would unnecessarily relax the threads connecting the state variables in the constitutive model, resulting in a posterior that

\footnotetext{
${ }^{5}$ In such a scenario, the presence of the prior $p_{C}\left(\boldsymbol{C} \mid \boldsymbol{\theta}_{C}\right)$ in Equation (15) would be superfluous.
} 
is also artificially vague. To address this ill-posedness, we invoke an additional postulate of parsimony, i.e. that the constitutive law is correct, unless strong evidence in the data/observables suggests differently. This suggests that, ceteris paribus, solutions with more constitutive law residuals $\boldsymbol{r}_{c}^{\left(i_{c}\right)}$ in Equation (9) being zero, should be favored. To that end, we advocate the use of Automatic Relevance Determination (ARD, [67, 102]), which is a heavy-tailed, hierarchical prior model, independently for each $\Lambda_{i_{c}}$ of the following form:

$$
p\left(\Lambda_{i_{c}} \mid \alpha_{0}, \beta_{0}\right)=\operatorname{Gamma}\left(\Lambda_{i_{c}} ; \alpha_{0}, \beta_{0}\right)=\frac{\Gamma\left(\alpha_{0}\right)}{\beta_{0}^{\alpha_{0}}} \Lambda_{i_{c}}^{\alpha_{0}-1} e^{-\beta_{0} \Lambda_{i_{c}}}
$$

Very small values for $\alpha_{0}, \beta_{0}$ (in the numerical investigations $\alpha_{0}=\beta_{0}=10^{-10}$ was used) promote robust sparsity patterns [12].

The combination of the aforementioned hierarchical prior with the augmented posterior of Equation (18) yields the following density (based on Bayes' rule):

$$
\begin{aligned}
p\left(\boldsymbol{\Sigma}, \boldsymbol{C}, \boldsymbol{U}_{i}, \boldsymbol{\Lambda} \mid \boldsymbol{u}_{o b s}, \boldsymbol{U}_{b}=\boldsymbol{u}_{0}\right) & =\frac{p\left(\boldsymbol{u}_{o b s} \mid \boldsymbol{\Sigma}, \boldsymbol{C}, \boldsymbol{U}_{i}, \boldsymbol{\Lambda}, \boldsymbol{U}_{b}=\boldsymbol{u}_{0}\right) p\left(\boldsymbol{\Sigma}, \boldsymbol{C}, \boldsymbol{U}_{i}, \boldsymbol{\Lambda} \mid \boldsymbol{U}_{b}=\boldsymbol{u}_{0}\right)}{p\left(\boldsymbol{u}_{o b s}\right)} \\
& =\frac{p\left(\boldsymbol{u}_{o b s} \mid \boldsymbol{U}_{i}\right) p\left(\boldsymbol{\Sigma}, \boldsymbol{C}, \boldsymbol{U}_{i} \mid \boldsymbol{\Lambda}, \boldsymbol{U}_{b}=\boldsymbol{u}_{0}\right) p(\boldsymbol{\Lambda})}{p\left(\boldsymbol{u}_{o b s}\right)}
\end{aligned}
$$

where $p\left(\boldsymbol{u}_{\text {obs }} \mid \boldsymbol{U}_{i}\right)$ is the aforementioned likelihood, $p(\boldsymbol{\Lambda})$ is the ARD defined in Equation (30) (we omit dependence on $\alpha_{0}, \beta_{0}$ for simplicity) and $p\left(\boldsymbol{\Sigma}, \boldsymbol{C}, \boldsymbol{U}_{i} \mid \boldsymbol{\Lambda}, \boldsymbol{U}_{b}=\boldsymbol{u}_{0}\right)$ can be found from the augmented prior of Equation (15) as:

$$
\begin{aligned}
p\left(\boldsymbol{\Sigma}, \boldsymbol{C}, \boldsymbol{U}_{i} \mid \boldsymbol{\Lambda}, \boldsymbol{U}_{b}=\boldsymbol{u}_{0}\right) & =\frac{p\left(\boldsymbol{\Sigma}, \boldsymbol{C}, \boldsymbol{U}_{i}, \boldsymbol{U}_{b}=\boldsymbol{u}_{0} \mid \boldsymbol{\Lambda}\right)}{p\left(\boldsymbol{U}_{b}=\boldsymbol{u}_{0} \mid \boldsymbol{\Lambda}\right)} \\
& =\frac{p\left(\boldsymbol{\Sigma}, \boldsymbol{C}, \boldsymbol{U}_{i}, \boldsymbol{U}_{b}=\boldsymbol{u}_{0} \mid \boldsymbol{\Lambda}\right)}{\int p\left(\boldsymbol{\sigma}, \boldsymbol{c}, \boldsymbol{u}_{i}, \boldsymbol{U}_{b}=\boldsymbol{u}_{0} \mid \boldsymbol{\Lambda}\right) d \boldsymbol{\sigma} d \boldsymbol{c} d \boldsymbol{u}_{i}} \\
& =\frac{\pi_{\boldsymbol{\Lambda}}\left(\boldsymbol{\Sigma}, \boldsymbol{C}, \boldsymbol{U}_{i}, \boldsymbol{U}_{b}=\boldsymbol{u}_{0}\right)}{\int \pi_{\boldsymbol{\Lambda}}\left(\boldsymbol{\sigma}, \boldsymbol{c}, \boldsymbol{u}_{i}, \boldsymbol{U}_{b}=\boldsymbol{u}_{0}\right) d \boldsymbol{\sigma} d \boldsymbol{c} d \boldsymbol{u}_{i}} .
\end{aligned}
$$

We discuss suitable inference tools based on Stochastic Variational Inference in the next section. 


\section{Variational Inference}

In the previous section, a probabilistic reformulation of the forward and inverse problems for linear elastostatics was proposed. The solution of canonical such problems amounts to inference tasks with respect to appropriate densities such as the ones in Equation (29) and Equation (32), over the unknown state variables and model parameters. While these densities do not require calls to any black-box, PDE solver, they are analytically intractable. We advocate the use of Variational Inference (VI) $[6,11]$ techniques, which, in contrast to Monte Carlo-based ones, are approximate albeit highly efficient. Such methods have risen into prominence for probabilistic inference tasks in the machine learning community $[2,55,83,100]$. They yield approximations to the target density, which are obtained by solving an optimization problem over a family of appropriately selected probability densities with the objective of minimizing the Kullback-Leibler divergence [23] (subsections 3.1 and 3.2). The success of such an approach hinges upon the selection of appropriate densities that have the capacity of providing good approximations while enabling efficient optimization with regards to their parameters (subsection 3.3). In the sequel we discuss the application of VI to the problems of interest and in the last subsection 3.4 present the proposed algorithms.

\subsection{VI for forward problems}

We first review the basic aspects of the method in the context of solving a canonical forward problem as described in section 2.3, which reduces to the density in Equation (29). For economy of notation we denote with $\boldsymbol{Y}$ the latent variables and by $\boldsymbol{V}$ the given/observed ones. Hence, in this case $\boldsymbol{Y}=\left\{\boldsymbol{\Sigma}, \boldsymbol{U}_{i}\right\}$ and $\boldsymbol{V}=\left\{\boldsymbol{C}, \boldsymbol{U}_{b}\right\}$. Let also $\boldsymbol{v}$ denote the given/observed value of $\boldsymbol{V}$, which according to Equation (29) is $\boldsymbol{v}=\left\{\boldsymbol{c}, \boldsymbol{u}_{0}\right\}$. The goal is to approximate the intractable density:

$$
p(\boldsymbol{Y} \mid \boldsymbol{V}=\boldsymbol{v}, \boldsymbol{\lambda})=\frac{\pi_{\boldsymbol{\lambda}}(\boldsymbol{Y}, \boldsymbol{V}=\boldsymbol{v})}{\int \pi_{\boldsymbol{\lambda}}(\boldsymbol{Y}, \boldsymbol{V}=\boldsymbol{v}) d \boldsymbol{Y}}
$$

Let $q(\boldsymbol{Y} ; \boldsymbol{\phi})$ a family of approximating densities parametrized by $\boldsymbol{\phi}$. We seek to determine the best possible approximation to $p(\boldsymbol{Y} \mid \boldsymbol{V}=\boldsymbol{v}, \boldsymbol{\lambda})$ by:

$$
\min _{\phi} K L(q(\boldsymbol{Y} ; \boldsymbol{\phi}) \| p(\boldsymbol{Y} \mid \boldsymbol{V}=\boldsymbol{v}, \boldsymbol{\lambda}))
$$


By employing Jensen's inequality one can establish that:

$$
\begin{aligned}
0 \leq K L(q(\boldsymbol{Y} ; \boldsymbol{\phi}) \| p(\boldsymbol{Y} \mid \boldsymbol{V}=\boldsymbol{v}, \boldsymbol{\lambda}))= & \int q(\boldsymbol{y} ; \boldsymbol{\phi}) \log \frac{q(\boldsymbol{y} ; \boldsymbol{\phi})}{p(\boldsymbol{y} \mid \boldsymbol{V}=\boldsymbol{v}, \boldsymbol{\lambda})} d \boldsymbol{y} \\
= & \int q(\boldsymbol{y} ; \boldsymbol{\phi}) \log \frac{q(\boldsymbol{y} ; \boldsymbol{\phi})}{\frac{\pi_{\boldsymbol{\lambda}}(\boldsymbol{y}, \boldsymbol{V}=\boldsymbol{v})}{\int \pi_{\boldsymbol{\lambda}}(\boldsymbol{y}, \boldsymbol{V}=\boldsymbol{v}) d \boldsymbol{y}}} d \boldsymbol{y} \\
= & \int q(\boldsymbol{y} ; \boldsymbol{\phi}) \log \frac{q(\boldsymbol{y} ; \boldsymbol{\phi})}{\pi_{\boldsymbol{\lambda}}(\boldsymbol{y}, \boldsymbol{V}=\boldsymbol{v})} d \boldsymbol{y} \\
& +\log \int \pi_{\boldsymbol{\lambda}}(\boldsymbol{y}, \boldsymbol{V}=\boldsymbol{v}) d \boldsymbol{y} \\
= & -\mathcal{F}_{\text {for }}(q(\boldsymbol{y} ; \boldsymbol{\phi}))+\log \int \pi_{\boldsymbol{\lambda}}(\boldsymbol{y}, \boldsymbol{V}=\boldsymbol{v}) d \boldsymbol{y} .
\end{aligned}
$$

Hence, minimizing the KL-divergence is equivalent to maximizing the Evidence Lower Bound $(\mathrm{ELBO},[13]) \mathcal{F}_{\text {for }}(q(\boldsymbol{y} ; \boldsymbol{\phi})) \leq \log \int \pi_{\boldsymbol{\lambda}}(\boldsymbol{y}, \boldsymbol{V}=\boldsymbol{v}) d \boldsymbol{y}$.

Based on the form of $\pi_{\lambda}$ (Equation (27)) one can obtain the following expression for the ELBO:

$$
\begin{aligned}
\mathcal{F}_{f o r}(q(\boldsymbol{y} ; \boldsymbol{\phi}))= & \int q(\boldsymbol{y} ; \boldsymbol{\phi}) \log \frac{\pi_{\boldsymbol{\lambda}}(\boldsymbol{y}, \boldsymbol{V}=\boldsymbol{v})}{q(\boldsymbol{y} ; \boldsymbol{\phi})} d \boldsymbol{y} \\
= & \mathbb{E}_{q(\boldsymbol{y} ; \boldsymbol{\phi})}\left[\log \psi_{1}(\boldsymbol{\sigma})\right]+\mathbb{E}_{q(\boldsymbol{y} ; \boldsymbol{\phi})}\left[\log \psi_{2}\left(\boldsymbol{\sigma}, \boldsymbol{u}_{i}, \boldsymbol{C}=\boldsymbol{c}, \boldsymbol{U}_{b}=\boldsymbol{u}_{0} ; \boldsymbol{\lambda}\right)\right] \\
& +\mathbb{E}_{q(\boldsymbol{y} ; \boldsymbol{\phi})}\left[\log \psi_{4}(\boldsymbol{\sigma})\right]+\mathbb{E}_{q(\boldsymbol{y} ; \boldsymbol{\phi})}\left[\log \psi_{5}\left(\boldsymbol{u}_{i}\right)\right]+\mathbb{H}[q(\boldsymbol{y} ; \boldsymbol{\phi})] \\
= & \mathcal{L}_{\text {for }}(\boldsymbol{\phi}),
\end{aligned}
$$

where $\mathbb{H}[q(\boldsymbol{y} ; \boldsymbol{\phi})]$ is the entropy of $q$. The term corresponding to $\psi_{3}(\boldsymbol{C})$ was omitted as it does not depend on $q$. We discuss the form of the $q$ employed in subsection 3.3 and the associated algorithmic steps (Algorithm 1) as well as provide additional details for the derivatives of the objective $\mathcal{L}_{\text {for }}$ with respect to $\phi$ in A.1.

\subsection{VI for inverse problems}

We consider now the application of Variational Inference for the solution of the inverse problem, as reformulated in section 2.3. This involves the augmented posterior of Equation (32). We denote again with $\boldsymbol{Y}$ the latent state variables which in this case consist of $\left\{\boldsymbol{\Sigma}, \boldsymbol{C}, \boldsymbol{U}_{i}\right\}$. The observed/known state variables $\boldsymbol{V}$ entail only $\boldsymbol{U}_{b}$. Furthermore, the model parameters $\boldsymbol{\Lambda}$ controlling constitutive model errors are unknown. Due to the undirected nature $[73,93,94,101]$ of the proposed graphical model and the dependence of the normalization constant $Z$ on $\boldsymbol{\Lambda}$ (Equation (28)), we adopt a hybrid strategy where the full posterior of $\boldsymbol{Y}$ is approximated and point-estimates, corresponding to the Maximum-A-Posteriori (MAP) value, for $\boldsymbol{\Lambda}$ are computed.

In particular, we consider the marginal posterior $p\left(\boldsymbol{\Lambda} \mid \boldsymbol{V}=\boldsymbol{v}, \boldsymbol{u}_{\text {obs }}\right)$ of $\boldsymbol{\Lambda}$ and seek to maximize it using a Variational Expectation-Maximization scheme [6] that is based 
on the following lower-bound:

$$
\begin{aligned}
\log p\left(\boldsymbol{\lambda} \mid \boldsymbol{U}_{b}=\boldsymbol{u}_{0}, \boldsymbol{u}_{o b s}\right) & =\log \int p\left(\boldsymbol{y}, \boldsymbol{\lambda} \mid \boldsymbol{U}_{b}=\boldsymbol{u}_{0}, \boldsymbol{u}_{o b s}\right) d \boldsymbol{y} \\
& =\log \int q(\boldsymbol{y} ; \boldsymbol{\phi}) \frac{p\left(\boldsymbol{y}, \boldsymbol{\lambda} \mid \boldsymbol{U}_{b}=\boldsymbol{u}_{0}, \boldsymbol{u}_{o b s}\right)}{q(\boldsymbol{y} ; \boldsymbol{\phi})} d \boldsymbol{y} \\
& \geq \int q(\boldsymbol{y} ; \boldsymbol{\phi}) \log \frac{p\left(\boldsymbol{y}, \boldsymbol{\lambda} \mid \boldsymbol{U}_{b}=\boldsymbol{u}_{0}, \boldsymbol{u}_{o b s}\right)}{q(\boldsymbol{y} ; \boldsymbol{\phi})} d \boldsymbol{y} \quad \text { (from Jensen's inequality) } \\
& =\mathcal{F}_{i n v}(q(\boldsymbol{y} ; \boldsymbol{\phi}), \boldsymbol{\lambda}),
\end{aligned}
$$

where $q(\boldsymbol{y} ; \boldsymbol{\phi})$ is a density with respect to the latent state variables. It can be readily shown [11] that the optimal $q^{o p t}$, for which the inequality above becomes an equality, corresponds to the posterior $p\left(\boldsymbol{y} \mid \boldsymbol{\lambda}, \boldsymbol{U}_{b}=\boldsymbol{u}_{o}, \boldsymbol{u}_{o b s}\right)$. The latter does not necessarily belong to the family of approximating densities $q(\boldsymbol{y} ; \boldsymbol{\phi})$ selected. Nevertheless, it suggests the following iterative scheme, where one alternates (until convergence) between the steps:

- $\mathbf{E}$ (xpectation)-step: The model parameters $\boldsymbol{\lambda}$ are fixed, and $\mathcal{F}_{i n v}$ is maximized with respect to $\boldsymbol{\phi}$ (so as it approximates as close as possible $\log p\left(\boldsymbol{\lambda} \mid \boldsymbol{U}_{b}=\right.$ $\left.\left.\boldsymbol{u}_{0}, \boldsymbol{u}_{o b s}\right)\right)$.

- $\mathbf{M}$ (aximization)-step: The parameters $\boldsymbol{\phi}$, and therefore $q$, remain fixed and $\mathcal{F}_{\text {inv }}$ is maximized with respect to the parameters $\boldsymbol{\lambda}$.

As with all Expectation-Maximization schemes [31, 75] several relaxations of the aforementioned steps are possible such as improving, rather than maximizing, $\mathcal{F}_{i n v}$ during any of the steps, partial updates of the parameters, etc.

Before we present the algorithmic details, we look more closely at the terms involved in $\mathcal{F}_{\text {inv }}$. In particular, based on Equations (31) and (32), we obtain:

$$
\begin{aligned}
\mathcal{F}_{i n v}(q(\boldsymbol{y} ; \boldsymbol{\phi}), \boldsymbol{\lambda})= & \int q(\boldsymbol{y} ; \boldsymbol{\phi}) \log \frac{p\left(\boldsymbol{y}, \boldsymbol{\lambda} \mid \boldsymbol{U}_{b}=\boldsymbol{u}_{0}, \boldsymbol{u}_{o b s}\right)}{q(\boldsymbol{y} ; \boldsymbol{\phi})} d \boldsymbol{y} \\
= & \int q(\boldsymbol{y} ; \boldsymbol{\phi}) \log \frac{p\left(\boldsymbol{u}_{o b s} \mid \boldsymbol{U}_{i}\right) p\left(\boldsymbol{\sigma}, \boldsymbol{c}, \boldsymbol{u}_{i} \mid \boldsymbol{\lambda}, \boldsymbol{U}_{b}=\boldsymbol{u}_{0}\right) p(\boldsymbol{\lambda})}{p\left(\boldsymbol{u}_{o b s}\right) q(\boldsymbol{y} ; \boldsymbol{\phi})} d \boldsymbol{\sigma} d \boldsymbol{c} d \boldsymbol{u}_{i} \\
= & \int q(\boldsymbol{y} ; \boldsymbol{\phi}) \log \frac{p\left(\boldsymbol{u}_{o b s} \mid \boldsymbol{U}_{i}\right)}{q(\boldsymbol{y} ; \boldsymbol{\phi})} d \boldsymbol{y}+\mathbb{E}_{q(\boldsymbol{y} ; \boldsymbol{\phi})}\left[\log p\left(\boldsymbol{\sigma}, \boldsymbol{c}, \boldsymbol{u}_{i} \mid \boldsymbol{\lambda}, \boldsymbol{U}_{b}=\boldsymbol{u}_{0}\right)\right] \\
& +\log p(\boldsymbol{\lambda})-\log p\left(\boldsymbol{u}_{o b s}\right) \\
= & \mathbb{E}_{q(\boldsymbol{y} ; \boldsymbol{\phi})}\left[\log p\left(\boldsymbol{u}_{o b s} \mid \boldsymbol{U}_{i}\right)\right]+\mathbb{H}[q(\boldsymbol{y} ; \boldsymbol{\phi})] \\
& +\mathbb{E}_{q(\boldsymbol{y} ; \boldsymbol{\phi})}\left[\log \frac{\pi_{\boldsymbol{\lambda}}\left(\boldsymbol{\sigma}, \boldsymbol{c}, \boldsymbol{u}_{i}, \boldsymbol{U}_{b}=\boldsymbol{u}_{0}\right)}{\left.\int \pi_{\boldsymbol{\lambda}}\left(\boldsymbol{\sigma}, \boldsymbol{c}, \boldsymbol{u}_{i}, \boldsymbol{U}_{b}=\boldsymbol{u}_{0}\right) d \boldsymbol{\sigma} d \boldsymbol{c} d \boldsymbol{u}_{i}\right]}\right. \\
& +\log p(\boldsymbol{\lambda}) \\
= & \mathbb{E}_{q(\boldsymbol{y} ; \boldsymbol{\phi})}\left[\log p\left(\boldsymbol{u}_{o b s} \mid \boldsymbol{U}_{i}\right)\right]+\mathbb{H}[q(\boldsymbol{y} ; \boldsymbol{\phi})] \\
& +\mathbb{E}_{q(\boldsymbol{y} ; \boldsymbol{\phi})}\left[\log \pi_{\boldsymbol{\lambda}}\left(\boldsymbol{\sigma}, \boldsymbol{c}, \boldsymbol{u}_{i}, \boldsymbol{U}_{b}=\boldsymbol{u}_{0}\right)\right]-\log \left[\int \pi_{\boldsymbol{\lambda}}\left(\boldsymbol{\sigma}, \boldsymbol{c}, \boldsymbol{u}_{i}, \boldsymbol{U}_{b}=\boldsymbol{u}_{0}\right) d \boldsymbol{\sigma} d \boldsymbol{c} d \boldsymbol{u}_{i}\right] \\
& +\log p(\boldsymbol{\lambda}),
\end{aligned}
$$

where we have omitted terms (e.g. $\left.\log p\left(\boldsymbol{u}_{\text {obs }}\right)\right)$ that do not depend on $q$ nor $\boldsymbol{\lambda}$. The most involved term in the expression above pertains to $\int \pi_{\lambda}\left(\boldsymbol{\sigma}, \boldsymbol{c}, \boldsymbol{u}_{i}, \boldsymbol{U}_{b}=\right.$ 
$\left.\boldsymbol{u}_{0}\right) d \boldsymbol{\sigma} d \boldsymbol{c} d \boldsymbol{u}_{i}$, which in principle needs to be determined in order to compute derivatives with respect to $\boldsymbol{\lambda}$. To that end, we advocate the use of an additional variational approximation employing a different class of densities denoted by $r(\boldsymbol{y} ; \boldsymbol{\xi})$ such that:

$$
\begin{aligned}
\log \left[\int \pi_{\boldsymbol{\lambda}}\left(\boldsymbol{\sigma}, \boldsymbol{c}, \boldsymbol{u}_{i}, \boldsymbol{U}_{b}=\boldsymbol{u}_{0}\right) d \boldsymbol{\sigma} d \boldsymbol{c} d \boldsymbol{u}_{i}\right] & =\log \int \pi_{\boldsymbol{\lambda}}\left(\boldsymbol{y}, \boldsymbol{U}_{b}=\boldsymbol{u}_{0}\right) d \boldsymbol{y} \\
& =\log \int r(\boldsymbol{y} ; \boldsymbol{\xi}) \frac{\pi_{\boldsymbol{\lambda}}\left(\boldsymbol{y}, \boldsymbol{U}_{b}=\boldsymbol{u}_{0}\right)}{r(\boldsymbol{y} ; \boldsymbol{\xi})} d \boldsymbol{y} \\
& \geq \int r(\boldsymbol{y} ; \boldsymbol{\xi}) \log \frac{\pi_{\boldsymbol{\lambda}}\left(\boldsymbol{y}, \boldsymbol{U}_{b}=\boldsymbol{u}_{0}\right)}{r(\boldsymbol{y} ; \boldsymbol{\xi})} d \boldsymbol{y} \\
& =\mathbb{E}_{r(\boldsymbol{y} ; \boldsymbol{\xi})}\left[\log \pi_{\boldsymbol{\lambda}}\left(\boldsymbol{y}, \boldsymbol{U}_{b}=\boldsymbol{u}_{0}\right)\right]+\mathbb{H}[r(\boldsymbol{y} ; \boldsymbol{\xi})] \\
& =\hat{\mathcal{F}}_{i n v}(r(\boldsymbol{y} ; \boldsymbol{\xi}), \boldsymbol{\lambda}) .
\end{aligned}
$$

We note again that the inequality above becomes an equality iff $r(\boldsymbol{y} ; \boldsymbol{\xi})=p\left(\boldsymbol{y}, \boldsymbol{U}_{b}=\right.$ $\boldsymbol{u}_{0}$ ) given in Equation (29). Unfortunately, using the lower bound $\hat{\mathcal{F}}_{\text {inv }}(r(\boldsymbol{y} ; \boldsymbol{\xi}), \boldsymbol{\lambda})$ in place of $\log \left[\int \pi_{\boldsymbol{\lambda}}\left(\boldsymbol{\sigma}, \boldsymbol{c}, \boldsymbol{u}_{i}, \boldsymbol{U}_{b}=\boldsymbol{u}_{0}\right) d \boldsymbol{\sigma} d \boldsymbol{c} d \boldsymbol{u}_{i}\right]$ in Equation (38) leads to an upper bound of $\mathcal{F}_{i n v}$ (due to the minus sign), i.e.:

$$
\begin{aligned}
\mathcal{F}_{i n v}(q(\boldsymbol{y} ; \boldsymbol{\phi}), \boldsymbol{\lambda}) \leq & \mathbb{E}_{q(\boldsymbol{y} ; \boldsymbol{\phi})}\left[\log p\left(\boldsymbol{u}_{o b s} \mid \boldsymbol{U}_{i}\right)\right]+\mathbb{H}[q(\boldsymbol{y} ; \boldsymbol{\phi})] \\
& +\mathbb{E}_{q(\boldsymbol{y} ; \boldsymbol{\phi})}\left[\log \pi_{\boldsymbol{\lambda}}\left(\boldsymbol{\sigma}, \boldsymbol{c}, \boldsymbol{u}_{i}, \boldsymbol{U}_{b}=\boldsymbol{u}_{0}\right)\right]-\hat{\mathcal{F}}_{i n v}(r(\boldsymbol{y} ; \boldsymbol{\xi}), \boldsymbol{\lambda}) \\
& +\log p(\boldsymbol{\lambda}) \\
= & \tilde{\mathcal{F}}_{i n v}(q(\boldsymbol{y} ; \boldsymbol{\phi}), r(\boldsymbol{y} ; \boldsymbol{\xi}), \boldsymbol{\lambda}) \\
= & \mathcal{L}_{i n v}(\boldsymbol{\phi}, \boldsymbol{\xi}, \boldsymbol{\lambda})
\end{aligned}
$$

Hence, $\mathcal{L}_{i n v}$ will not necessarily be a lower bound of the marginal log-posterior $\log p\left(\boldsymbol{\lambda} \mid \boldsymbol{U}_{b}=\boldsymbol{u}_{0}, \boldsymbol{u}_{\text {obs }}\right)$ in Equation (37). This introduces an additional error, which is smaller the closer $r(\boldsymbol{y} ; \boldsymbol{\xi})$ is to the optimum. We note that the final objective $\tilde{\mathcal{F}}_{\text {inv }}$ depends on two sets of approximating densities: a) $q(\boldsymbol{y} ; \boldsymbol{\phi})$ that attempts to approximate the posterior (Equation (37)), and b) $r(\boldsymbol{y} ; \boldsymbol{\xi})$ which attempts to approximate the prior (Equation (39)). As a result, the E-step in the Variational EM scheme described previously should involve (with $\boldsymbol{\lambda}$ fixed), apart from the updates of $q(\boldsymbol{y} ; \boldsymbol{\phi})$, the maximization of $\hat{F}_{\text {inv }}$ (Equation (39)) with respect to $r(\boldsymbol{y} ; \boldsymbol{\phi})$.

We discuss the form of the approximating densities $q, r$ in subsection 3.3 and the associated algorithmic steps (Algorithms 2 and 3) as well as provide additional details for the derivatives of the objective $\mathcal{L}_{\text {for }}$ with respect to $\boldsymbol{\phi}, \boldsymbol{\xi}$ and $\boldsymbol{\lambda}$ in A.2.

\subsection{Form of approximating densities}

While arbitrary forms of approximating densities $q$ and $r$ are allowed, for reasons of computational efficiency we focus on the Gaussian family of distributions $\mathcal{N}(\boldsymbol{\mu}, \boldsymbol{S})$, parameterized by the mean $\boldsymbol{\mu}$ and covariance matrix $\boldsymbol{S}$, i.e $\boldsymbol{\phi}$ or $\boldsymbol{\xi}=\{\boldsymbol{\mu}, \boldsymbol{S}\}$. In terms of the covariance matrix, several parameterizations are possible:

- diagonal covariance: this mean-field assumption represents the most efficient choice, where all correlations between latent variables are neglected and $\boldsymbol{S}$ becomes a diagonal matrix. 
- full covariance: taking into account all possible cross-correlations, the covariance matrix can be parameterized using its Cholesky decomposition $\boldsymbol{S}=\boldsymbol{L} \boldsymbol{L}^{T}$.

- banded covariance: modeling only a few correlations that are assumed to dominate the variance can result in a banded covariance structure, exhibiting significantly less parameters than a full covariance model.

Diagonal covariance models scale linearly with the dimension of the latent variables, however, they tend to underestimate the posterior uncertainty. Full covariance models provide better uncertainty estimates, but scale quadratically with the dimension of the latent variables. As a compromise, we chose a banded covariance model and make use of the spatial character of the latent variables in order to endow a nearest-neighbor correlation structure. More specifically, we assume that off-diagonal entries in $\boldsymbol{S}$ are nonzero if they pertain to quantities associated with the same or a neighboring (i.e. with a common boundary) element. We provide numerical evidence on the relative accuracy of all three covariance models in section 4.1 .

\subsection{Stochastic Variational Inference}

The optimization objectives $\mathcal{L}_{\text {for }}$ and $\mathcal{L}_{\text {inv }}$ presented in subsections 3.1 and 3.2 for the Gaussian densities $q$ and $r$ parametrized as in subsection 3.3, is analytically intractable due to the implicit dependence on $\boldsymbol{\phi}$ and $\boldsymbol{\xi}$ of expectations involving $q$ and $r$ (see Equation (36) or Equation (40)). To that end we employ Stochastic Variational Inference (SVI) [51, 83, 98], which makes use of Monte Carlo estimates of the gradients of the objective functions in order to iteratively update the parameters $\boldsymbol{\phi}$ and $\boldsymbol{\xi}$ as well as $\boldsymbol{\lambda}$. Further details for the gradients are contained in A.1 and A.2.

We note that the use of Gaussian densities for $q$ and $r$ enables the application of the well-known reparametrization trick [60, 86, 98], which can significantly reduce the variance of the Monte Carlo estimators for the gradients. The latter consists of the simple observation that for any function $f(\boldsymbol{y})$ its expectation with respect to a Gaussian $q_{\phi}(y)=\mathcal{N}\left(\boldsymbol{y} \mid \boldsymbol{\mu}, \boldsymbol{S}=\boldsymbol{L} \boldsymbol{L}^{T}\right)$ can be re-expressed as:

$$
\mathbb{E}_{q(\boldsymbol{y} ; \phi)}[f(\boldsymbol{y})]=\mathbb{E}_{\mathcal{N}(\mathbf{0}, \boldsymbol{I})}[f(\boldsymbol{\mu}+\boldsymbol{L} \boldsymbol{\eta})],
$$

where the latter expectation is with respect to $\boldsymbol{\eta} \sim \mathcal{N}(\mathbf{0}, \boldsymbol{I})$ based on the transformation $\boldsymbol{y}=\boldsymbol{g}_{\phi}(\boldsymbol{\eta})=\boldsymbol{\mu}+\boldsymbol{L} \boldsymbol{\eta}$. Hence, derivatives with respect to $\boldsymbol{\phi}=\{\boldsymbol{\mu}, \boldsymbol{L}\}$ can be computed by employing the chain rule as follows:

$$
\nabla_{\phi} \mathbb{E}_{q(\boldsymbol{y} ; \phi)}[f(\boldsymbol{y})]=\mathbb{E}_{\mathcal{N}(\mathbf{0}, \boldsymbol{I})}\left[\nabla_{\boldsymbol{y}} f(\boldsymbol{\mu}+\boldsymbol{L} \boldsymbol{\eta}) \nabla_{\phi} \boldsymbol{g}_{\phi}(\boldsymbol{\eta})\right]
$$

We finally note that the gradient-based updates of the parameters are carried out using the Adam scheme [59] (A.3). We recap the basic algorithmic steps for the application of SVI for the solution of forward problems in Algorithm 1 and inverse problems in Algorithm 3 with references to the pertinent equations/sections. We 
have also included Algorithm 2, which can be employed for the solution of inverse problems in the absence of model errors (i.e. assuming $\boldsymbol{\lambda} \rightarrow \infty$ ). With regards to the overall scalability of the algorithms, we note that due to their first-order nature they scale linearly with the number of parameters $\boldsymbol{\phi}$ for forward problems and $(\boldsymbol{\phi}, \boldsymbol{\xi}, \boldsymbol{\lambda})$ for general inverse problems.
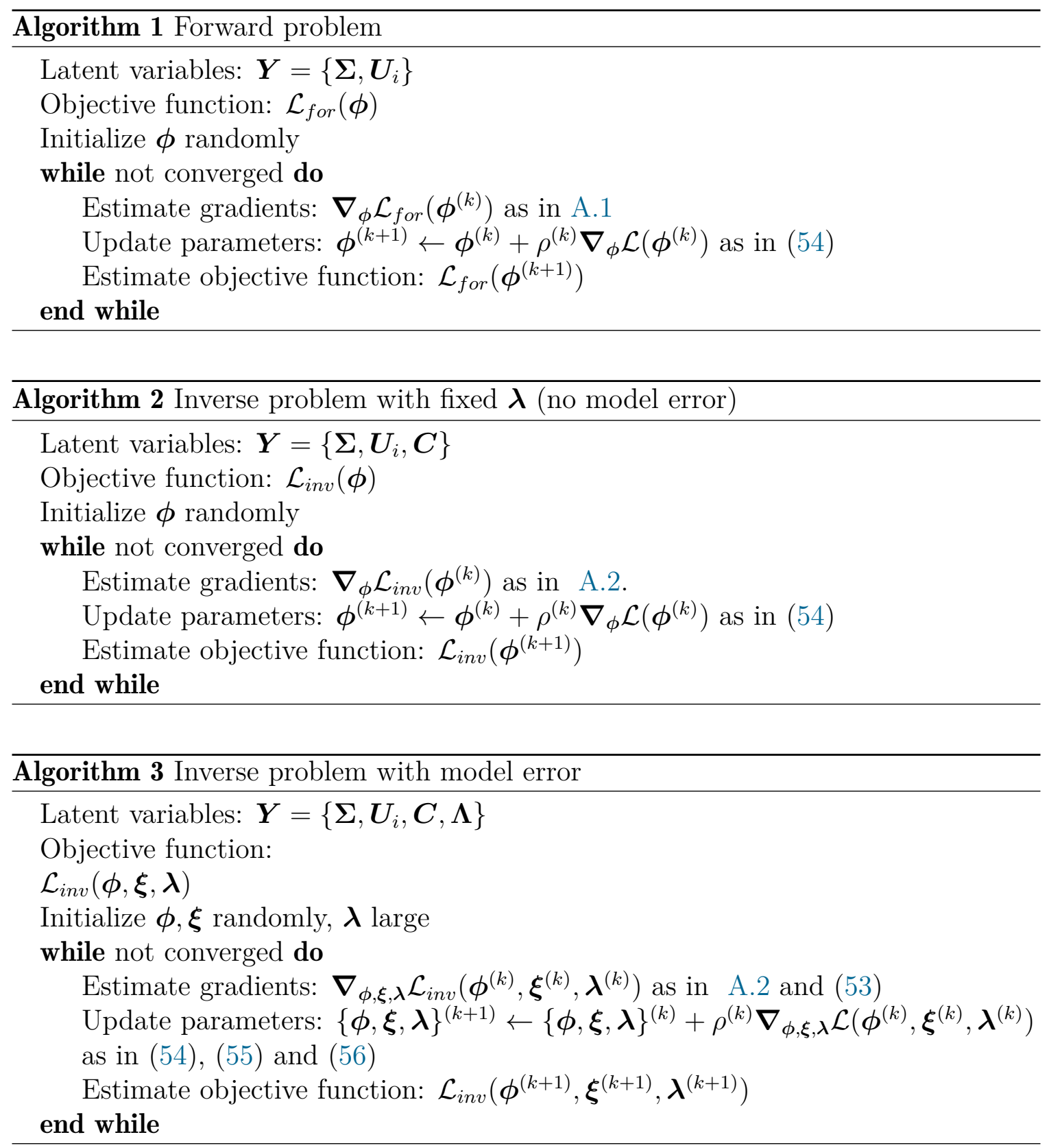


\section{Numerical illustrations}

In the following, we demonstrate the capabilities and efficacy of the proposed modeling framework for an elliptic problem described by the equations in section 2 and for the geometry and boundary conditions in Figure 4. The linear elastostatic problem that will be considered is motivated by an application to elastography, where the identification of tumors/inclusions in healthy tissue is sought.

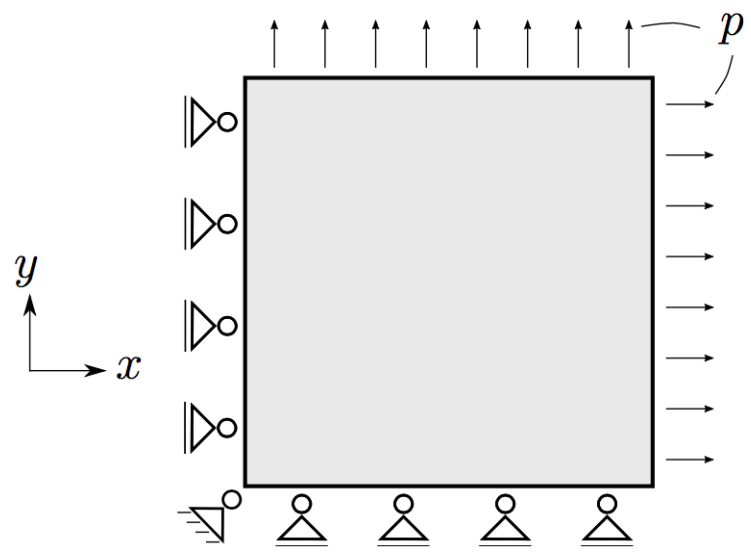

Figure 4: The two dimensional domain considered with dimensions $[0, L] \times[0, L]$, with $L=10$, thickness $d=1$ and Young's moduli $E(x, y)$. External normal tractions (Neumann boundary conditions) $p=1.0$ are applied along the boundaries at $x=L$ and $y=L$, while the boundaries at $x=0$ and $y=0$ are fixed in the respective normal direction.

We consider plane stress conditions and the only unknown parameter is assumed to be the Young's modulus ${ }^{6}$, the values of which at various points/elements are represented by the vector $\boldsymbol{C}$. In section 4.1 , we investigate comparatively the performance of the three covariance structures discussed in section 3.3 and demonstrate that the banded structure offers a good balance between accuracy and scalability. Subsequently, we investigate the following cases:

- in section 4.2 , the solution of a forward problem under the probabilistic inference reformulation proposed.

- in section 4.3 the solution of an inverse problem under the assumption of no model errors.

- in section 4.4, the solution of an inverse problem with model errors.

The goal is three-fold: a) to assess the validity of the model-informed augmented prior (section 2) in representing the governing equations, b) to assess the accuracy of the proposed inference schemes in identifying material parameters and their spatial variability, and c) to assess the ability of the proposed formulation to identify

\footnotetext{
${ }^{6} \mathrm{~A}$ Poisson ratio of $\nu=0.49$ was used to approximate incompressible material behavior
} 
and quantify model discrepancies. We note that the results obtained in the latter three subsections were based on a banded covariance structure. Parameters for the Adam stochastic optimization scheme are contained in Appendix A.3. For the inverse problems (sections 4.3,4.4), a total variation prior on the material parameters is employed, penalizing jumps in the material parameters between neighboring elements and corresponding to the factor potential

$$
\psi_{3}(\boldsymbol{C})=\prod_{e=1}^{n_{e l}} e^{-\frac{\theta_{C}}{2} \sum_{i \in \mathcal{N}\left(c_{e}\right)}\left|c_{e}-c_{i}\right|},
$$

with $\theta_{C}$ controlling the penalty. In terms of the hyperparameters for the factor potentials appearing in the augmented prior/posterior densities, we chose for the forward problems $\varepsilon=10^{-4}, \lambda=10^{8}, \theta_{u}=\theta_{\sigma}=\theta_{C}=10^{-6}$. For the inverse problems, these parameters need to be adjusted to the respective likelihood noise, e.g. for $\sigma_{n}=10^{-4}$ and for fixed model parameters, we chose $\varepsilon=10^{-4}, \Lambda=10^{8}, \theta_{u}=\theta_{\sigma}=$ $10^{-6}, \theta_{C}=10^{4}$.

\subsection{Comparison of diagonal, banded and full covariance approxima- tions}

Before the larger numerical examples are presented, we compare the accuracy of the proposed banded covariance model in relation to the diagonal and full covariance cases. For that purpose, a forward problem on a very small regular mesh with only eight triangular elements is considered as well as a uniform distribution of material parameters (i.e. $c_{e}=1.0$ over all elements). In this case, the number of latent variables is 42 (i.e. $\operatorname{dim}(\boldsymbol{Y})=42$ ) and consist of stresses $\boldsymbol{\Sigma}$ and displacements $\boldsymbol{U}_{i}$. In order to account for nearest-neighbor correlations, while minimizing the band-length and therefore the number of unknown parameters, a Cuthill-McKee permutation algorithm ${ }^{7}$ was used and its effect is illustrated in Figure 5. Therein, one can also see the number of non-zero terms of the Cholesky factor $\boldsymbol{L}$ that was ultimately used in the parametrization $\boldsymbol{\phi}$ (along with the mean $\boldsymbol{\mu}$ of the Gaussian).

Figure 6 depicts the estimated ELBO $\mathcal{L}_{\text {for }}$ (Equation (36)) with three covariance structures for $q$, i.e. diagonal (which requires 42 parameters), full (which requires 903 parameters) and banded (which requires 556 parameters as per Figure 5 ). The diagonal covariance model $\left(\mathcal{L}_{\text {for }} \approx-175.50\right)$ is clearly outperformed by the banded $\left(\mathcal{L}_{\text {for }} \approx-156.39\right)$ and full covariance $\left(\mathcal{L}_{\text {for }} \approx-156.2264\right)$ approximations. Given that the latter is the most accurate (at least in the class of Gaussian $q$ 's considered), we note that the banded covariance can achieve the same level of accuracy with much fewer parameters. This behavior is also reflected in the learned covariance matrices of the respective models, as illustrated in Figure 7. The diagonal covariance model clearly misses important correlations captured by the banded and full covariance variational

\footnotetext{
${ }^{7}$ The following implementation was used: https://de.mathworks.com/help/matlab/ref/ symrem.html
} 

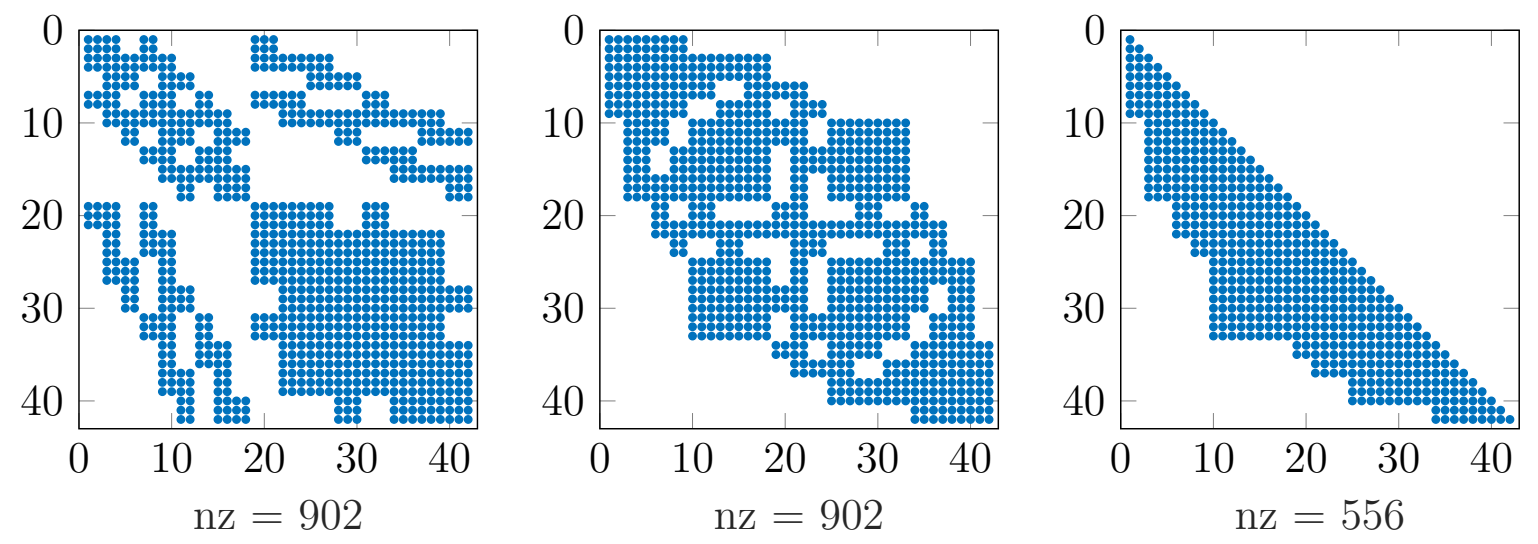

Figure 5: The left figure visualizes the covariance structure that accounts for nearestneighbor correlations (blue dots indicating a non-zero value) and requires 902 parameters. In the middle, this structure was reordered using a Cuthill-McKee permutation algorithm and on the right hand side, the final Cholesky factor $\boldsymbol{L}$ is shown with the number of non-zero parameters having been reduced to 556 .

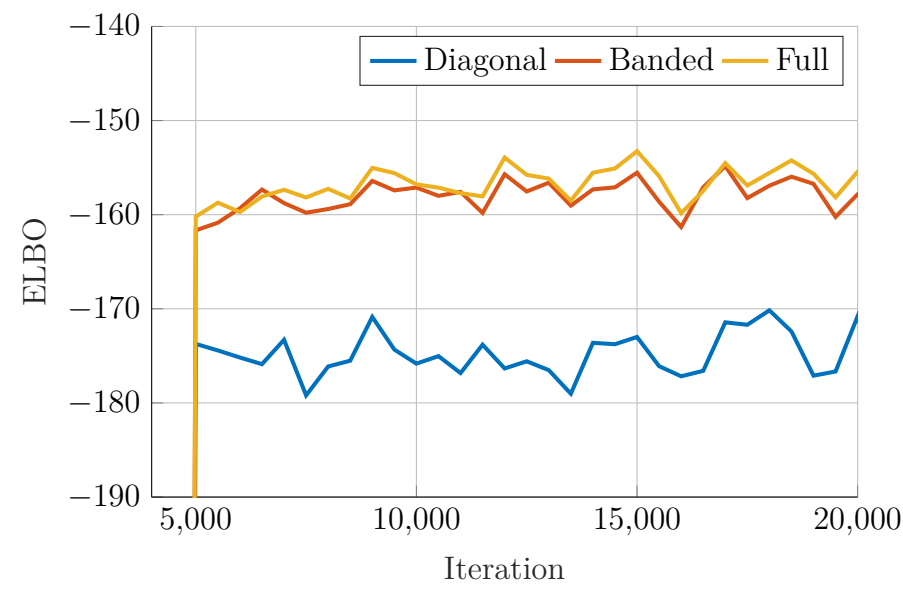

Figure 6: Estimated ELBO $\mathcal{L}_{\text {for }}$ over the number of iterations for the simple forward problem (section 4.1) and the three considered covariance models: diagonal $\left(\mathcal{L}_{\text {for }} \approx\right.$ $-175.50)$, banded $\left(\mathcal{L}_{\text {for }} \approx-156.39\right)$ and full covariance $\left(\mathcal{L}_{\text {for }} \approx-156.2264\right)$.

approximations. The latter two mostly differ in the learned cross-correlations $\boldsymbol{S}_{\boldsymbol{u}}$, while the displacement covariances $\boldsymbol{S}_{\boldsymbol{u} u}$ and $\boldsymbol{\Sigma}_{\sigma \sigma}$ are very similar.

Lastly, Figure 8 provides a graphical overview over the scalability of the three models. The number of optimization parameters of the banded covariance variational model scales approximately to the power of 1.5 with the number of latent variables. For comparison, the full covariance variational model scales to the power of 2 (quadratically), while the diagonal covariance scales linearly. 

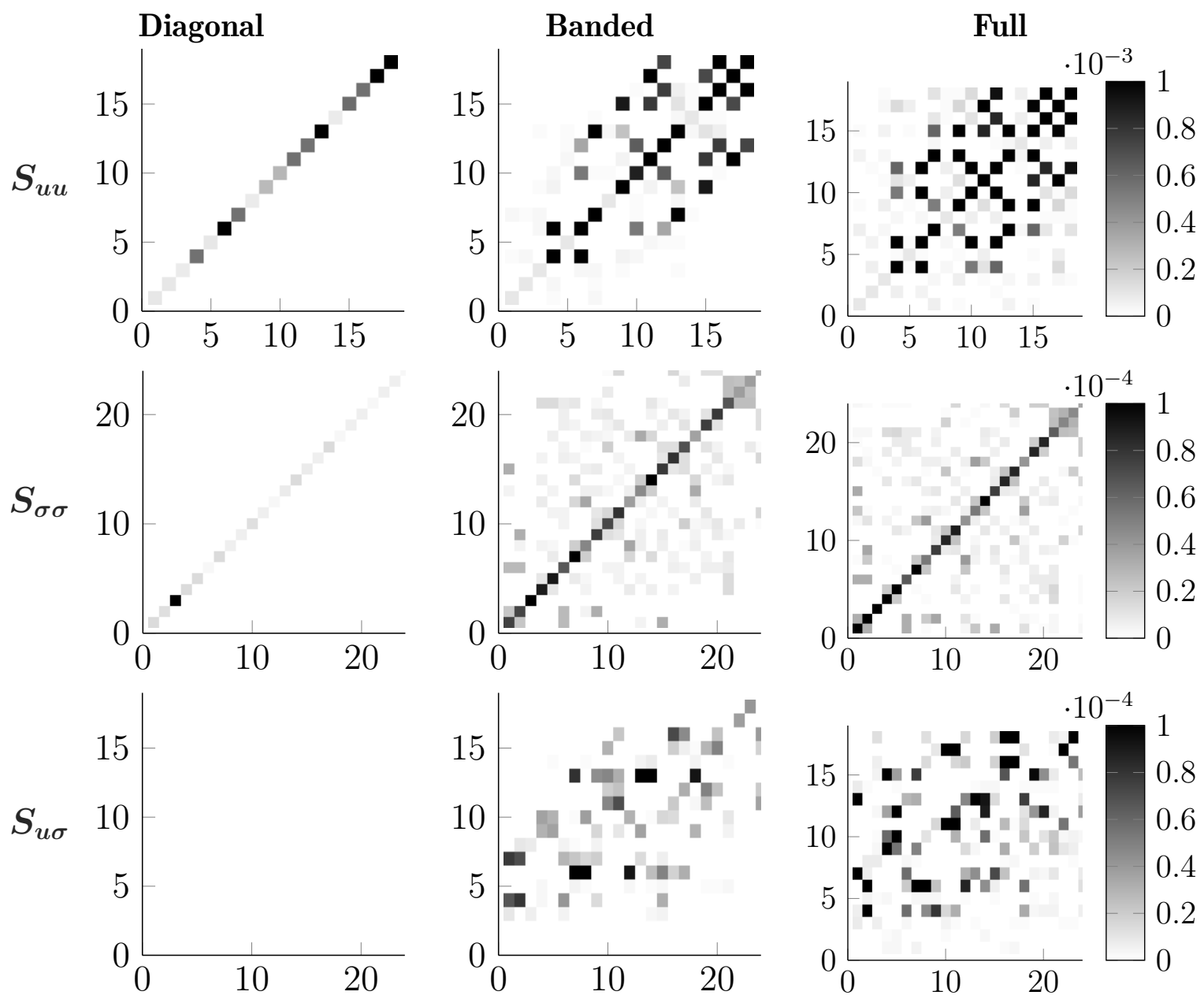

Figure 7: Learned covariance matrices $\boldsymbol{S}$ using the diagonal, banded and full covariance models for the test forward problem. For illustration purposes, the covariance matrix has been partitioned so that in the first row covariances $\boldsymbol{S}_{\boldsymbol{u}}$ associated with the displacements are depicted, in the second row the covariances between the stress variables $\boldsymbol{S}_{\sigma \sigma}$ and in the third row the cross-correlations $\boldsymbol{S}_{\boldsymbol{u} \boldsymbol{\sigma}}$ between the displacements and stresses.

\subsection{Forward problem}

The problem domain from Figure 4 is discretized using a randomized Delaunay triangulation $^{8}$ resulting in $n_{e l}=325$ elements, $n_{\text {nodes }}=184$ nodes. The corresponding number of latent variables (i.e. stresses $\boldsymbol{\Sigma}$ and displacements $\boldsymbol{U}_{i}$ ) was 1343 and the number of parameters $\boldsymbol{\phi}$ in the approximating density 146989 (i.e. $\operatorname{dim}(\boldsymbol{\mu})=1343$ and $\operatorname{dim}(\boldsymbol{L})=145646$ ). At the center of the physical domain, an inclusion was considered as shown in Figure 9. The spatial distribution of the Young's modulus was

\footnotetext{
${ }^{8}$ The Triangle software was used available from: https : / /www. cs. cmu . edu/ quake/triangle. html
} 


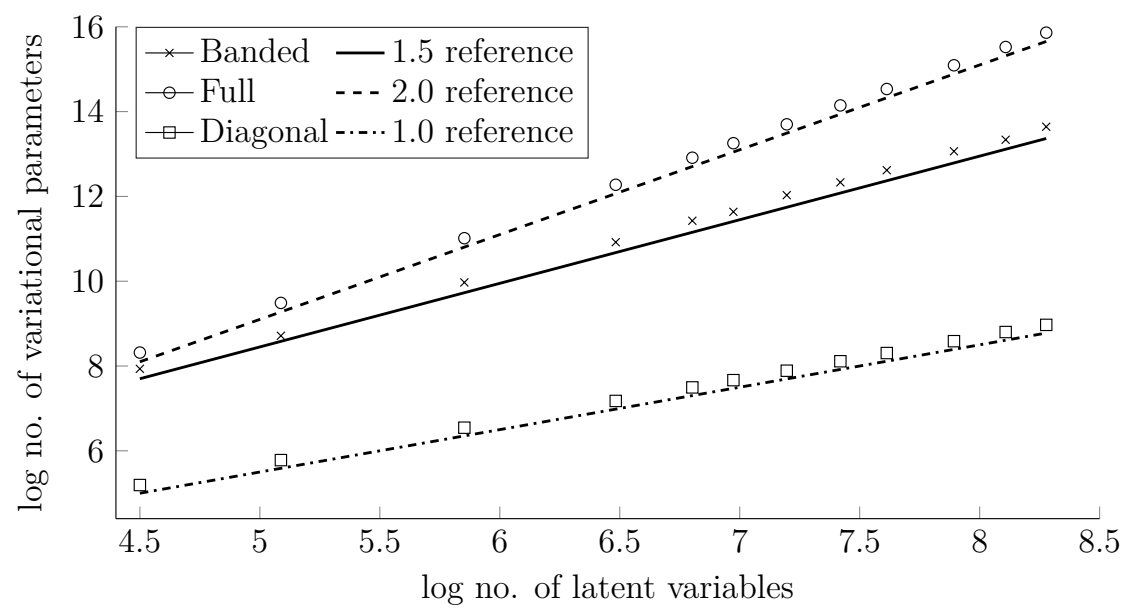

Figure 8: Number of variational parameters $\boldsymbol{\phi}$ needed for the covariance matrix $\boldsymbol{S}$ as a function of the latent variables $\boldsymbol{y}$. The number of optimization parameters of the banded covariance variational model scales approximately to the power of 1.5 with the number of latent variables. For comparison, the full covariance variational model scales to the power of 2 , while the diagonal covariance exhibits linear scaling.

assumed to be (if $\left(x_{e}, y_{e}\right)$ are the coordinates of the center of an element $e$ ):

$$
c_{e}= \begin{cases}0.2 & \text { if }\left(x_{e}-5\right)^{2}+\left(y_{e}-5\right)^{2} \leq 1 \\ 1.0 & \text { otherwise }\end{cases}
$$

The evolution of the objective function $\mathcal{L}_{\text {for }}$ over the iterations for the parameter updates is shown in Figure 9. As previously discussed, the output of the proposed formulation for such a forward problem is a density for the latent variables, namely stresses $\boldsymbol{\Sigma}$ and (interior) displacements $\boldsymbol{U}_{i}$. Two-dimensional plots of the means of these variables are contained in Figure 10. More importantly, in Figure 11 the means as well as the $95 \%$ credible intervals (CI) along the diagonal line from the lower left corner at $(x, y)=(0,0)$ to the upper right corner at $(x, y)=(10,10)$ are compared with the values (reference) one would obtain by solving deterministically the governing PDE using the same mesh/discretization. The excellent agreement between the two indicate that the proposed augmented prior model correctly encapsulates the governing equations and can be employed as suggested in section 2 instead of the black-box solver in the context of the ensuing inverse problems.

\subsection{Inverse problem without model error}

In this section we investigate the performance of the proposed formulation for an inverse problem without model error, i.e. assuming $\boldsymbol{\lambda} \rightarrow \infty$. To that end, we assume material properties as in Equation (44) and perform a forward analysis using a deterministic finite element solver which employed a different randomized mesh as compared to the one used for the inverse problem (Figure 9). The displacements 

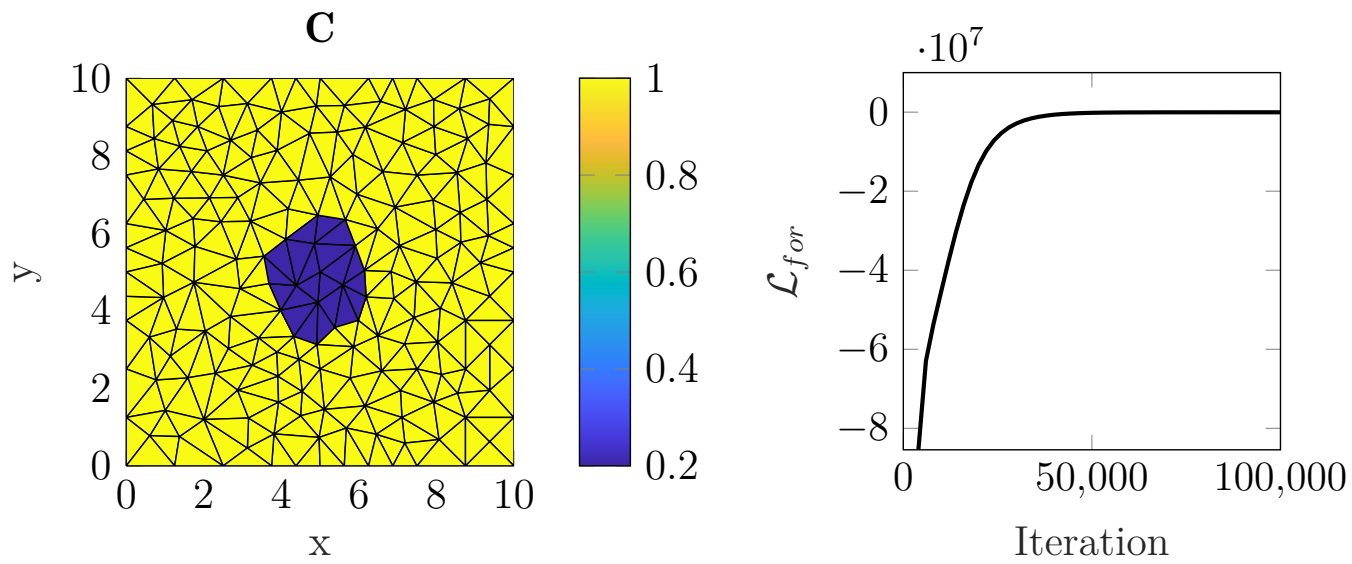

Figure 9: Spatial distribution of Young's modulus for the forward problem (left) and objective function $\mathcal{L}_{\text {for }}$ over the number of iterations (right).

computed were contaminated with various levels of additive Gaussian noise in order to obtain the observables $\boldsymbol{u}_{\text {obs }}$. The corresponding Signal to Noise Ratios (SNR) considered were $S N R_{d B}=60 d B, 50 d B$ and $40 d B$.

In such an inverse problem, the latent variables are of dimension 1668 and include stresses $\boldsymbol{\Sigma}$, (interior) displacements $\boldsymbol{U}_{i}$ as well as the material parameters $\boldsymbol{C}$. Using the banded covariance model gave rise to 226578 parameters $\boldsymbol{\phi}$. Indicative twodimensional plots for $S N R_{d B}=50 \mathrm{~dB}$ of the posterior means and variances for all latent fields/variables are contained in Figure 12 and Figure 13 respectively. In Figure 14, posterior means and 95\% confidence intervals along the diagonal are depicted and compared with the reference solutions. In all cases, good agreement is observed and the predicted uncertainty envelops the ground truth. The evolution of the ELBO objective $\mathcal{L}_{i n v}$ as well as convergence plots of selected optimization parameters are provided in Figure 15.

For comparison purposes, posterior statistics of the material parameters are depicted in Figure 16, corresponding to various SNRs. As expected, and as the noise level increases, the total variation prior for the material parameters plays an essential role as a regularizer. An important feature of the banded covariance variational approximation is that in all cases it is able to capture the truth in the $95 \%$ confidence interval, even when the noise becomes very large.

\subsection{Inverse problem with model error}

In this final problem scenario, we consider an inverse problem in the presence of model errors. We employed the same deterministic forward model described in the previous section with one fundamental difference with regards to the constitutive law for the 

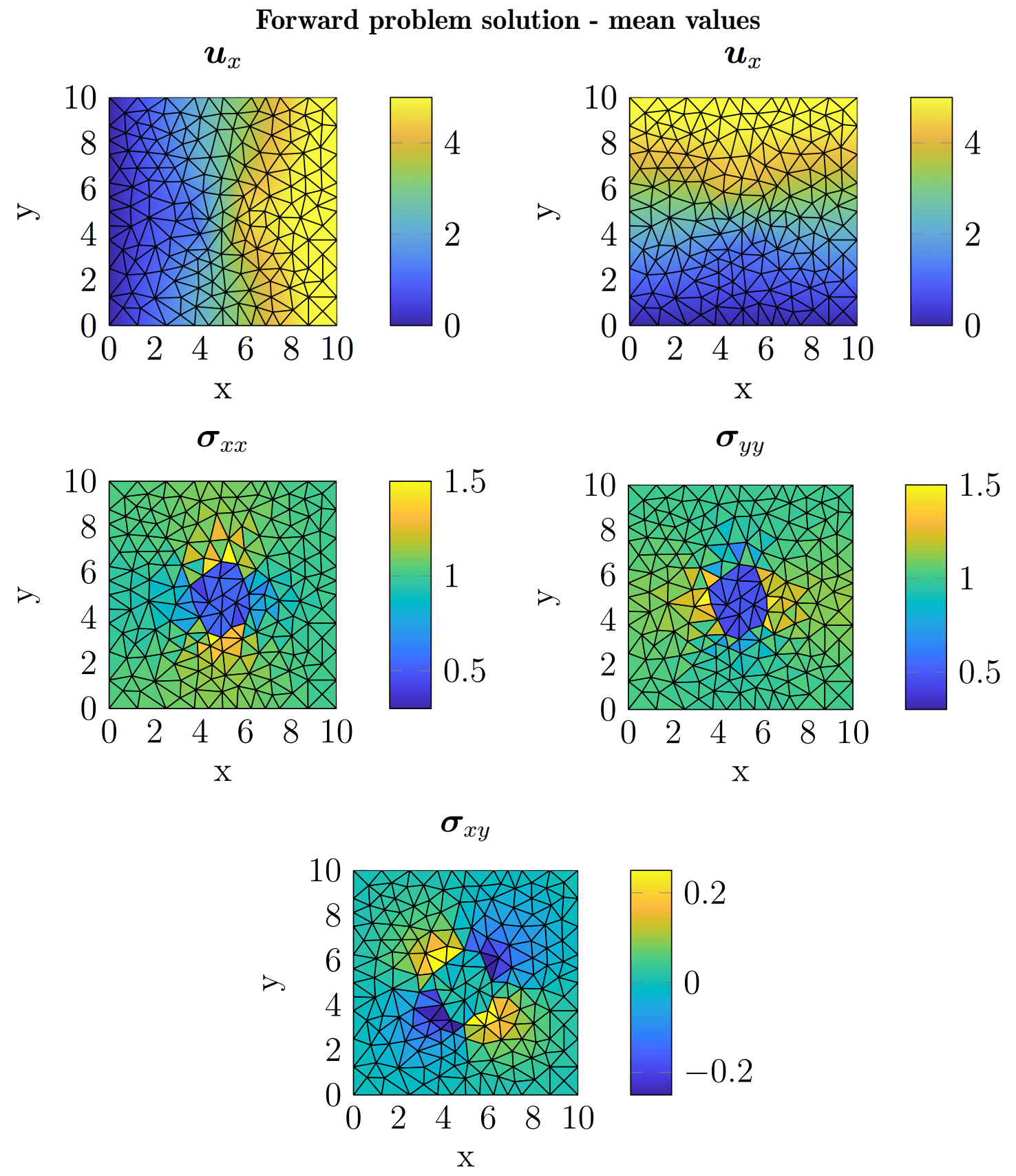

Figure 10: Inferred mean values for the displacements and stresses for the forward problem with a circular inclusion at the domain center. 

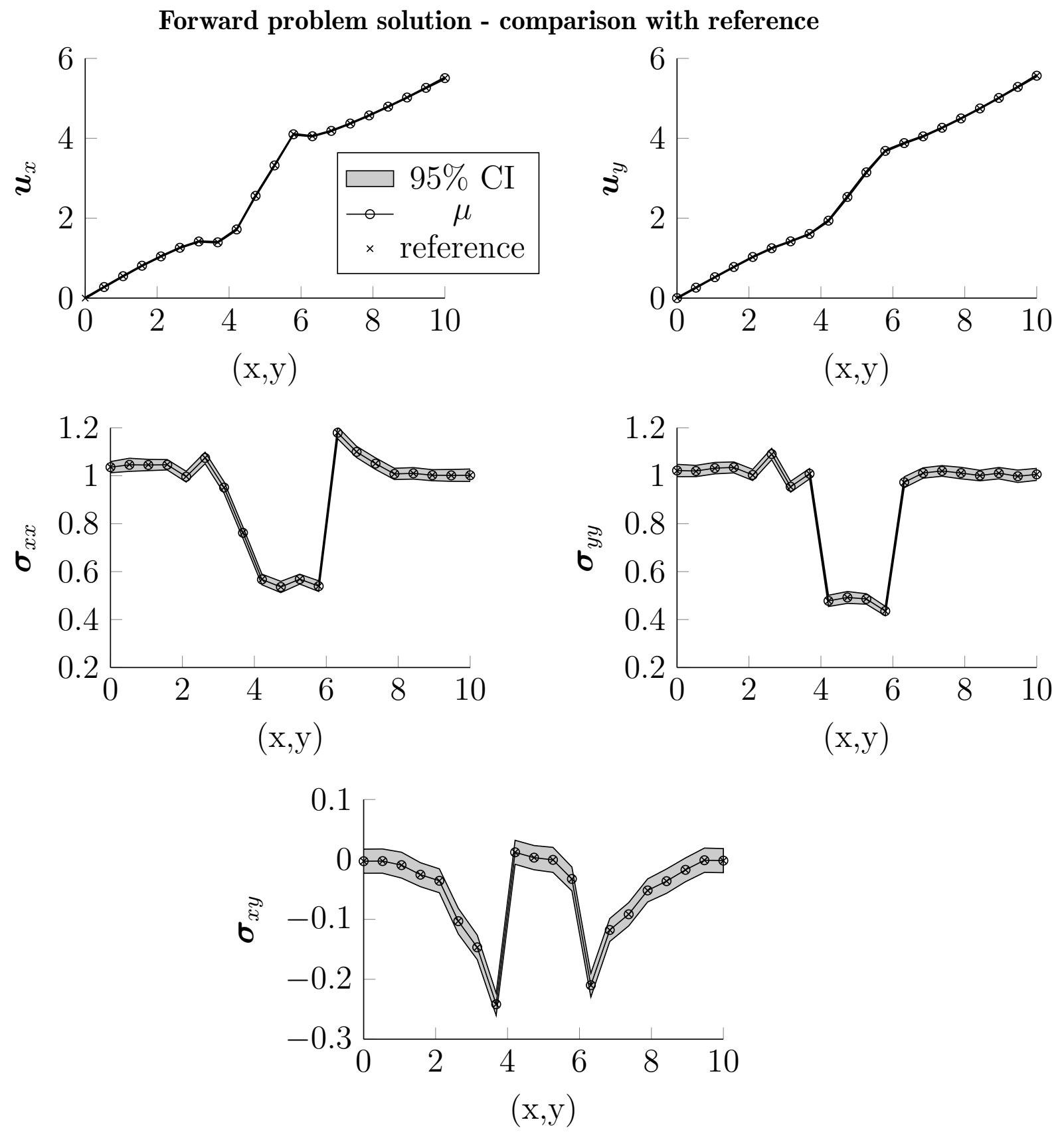

Figure 11: Comparison of reference displacements and stresses obtained from a deterministic FE model along the diagonal from $(0,0)$ to $(10,10)$ with the inferred means and $95 \%$ confidence intervals. 


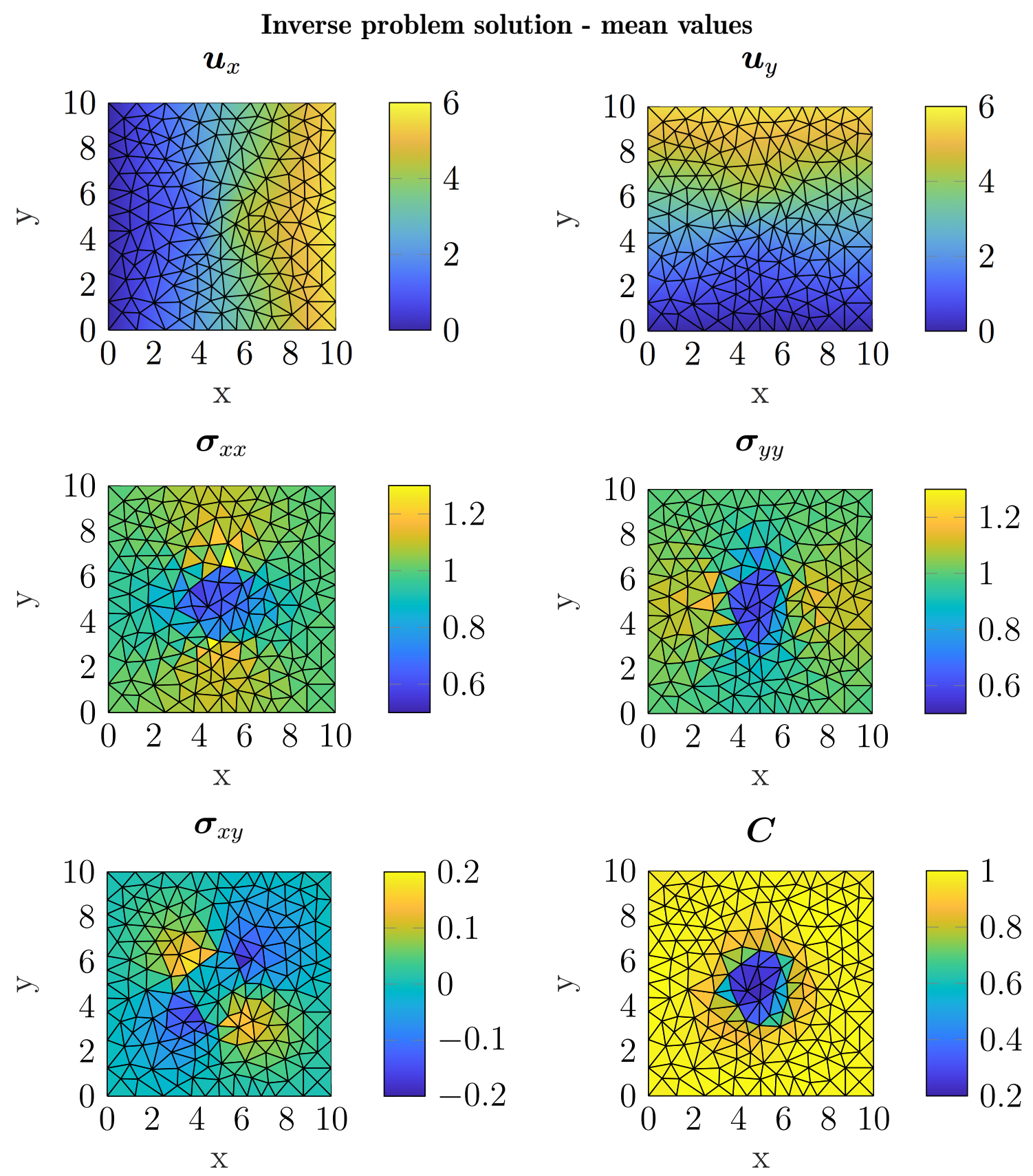

Figure 12: Inferred posterior mean values for displacements $u_{x}, u_{y}$, stresses $\sigma_{x x}, \sigma_{y y}, \sigma_{x y}$ and material parameters $\boldsymbol{C}$ for the inverse problem (without model error - section $4.3-S N R_{d B}=50 d B$ ). 

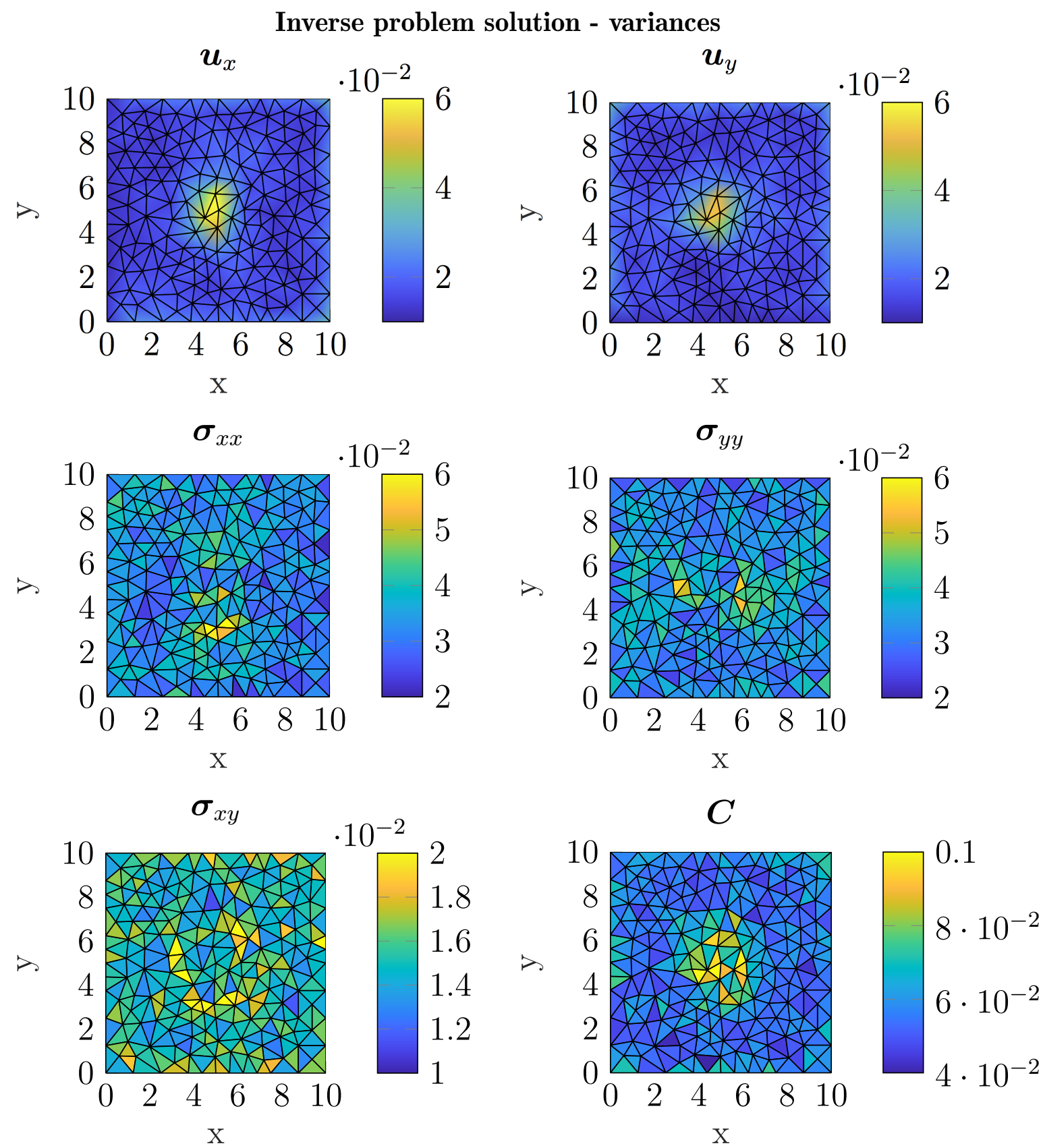

Figure 13: Inferred posterior variance values for displacements $u_{x}, u_{y}$, stresses $\sigma_{x x}, \sigma_{y y}, \sigma_{x y}$ and material parameters $\boldsymbol{C}$ for the inverse problem (without model error - section 4.3) - $S N R_{d B}=50 d B$ ). 

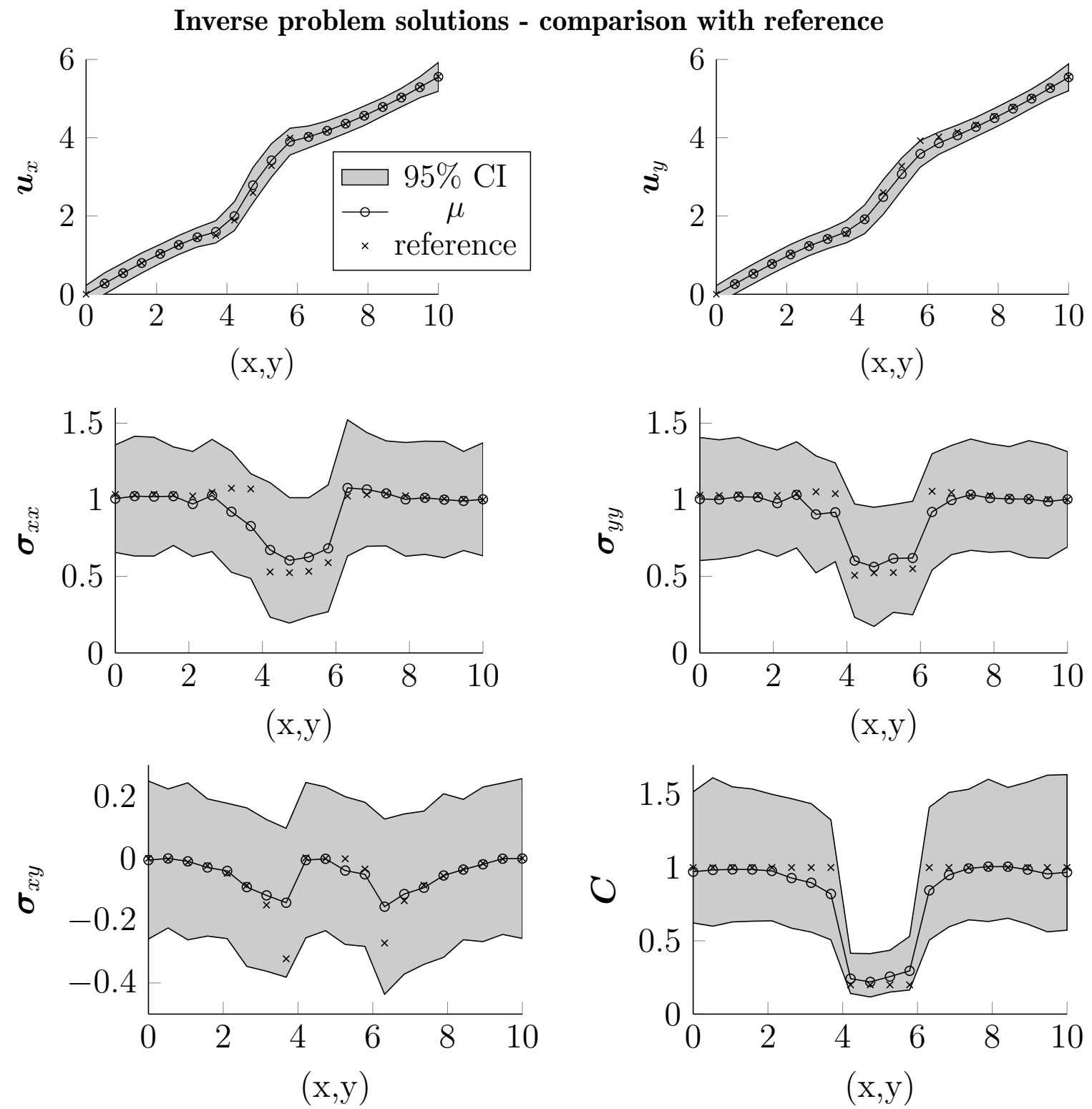

Figure 14: Comparison of reference values for displacements $u_{x}, u_{y}$, stresses $\sigma_{x x}, \sigma_{y y}, \sigma_{x y}$ and material parameters $\boldsymbol{C}$ along the diagonal (from $(0,0)$ to $(10,10)$ ) with the inferred posterior means and $95 \%$ confidence intervals $\left(S N R_{d B}=50 d B\right)$. 

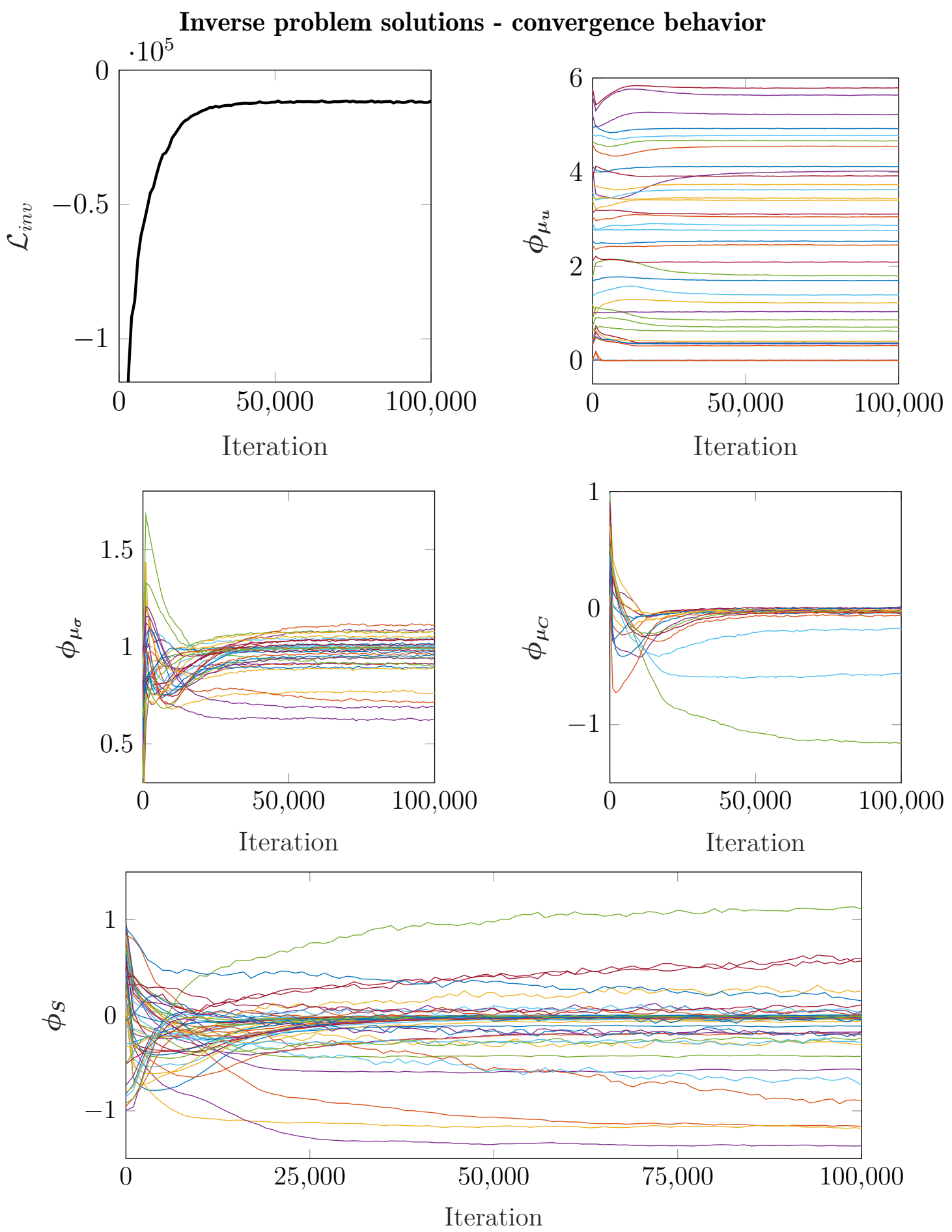

Figure 15: Evolution of the objective function $\mathcal{L}_{i n v}$ (upper left) and convergence behavior of the displacement means $\phi_{\mu_{u}}$ (upper right), stress means $\phi_{\mu_{\sigma}}$ (center left) and material parameter means $\phi_{\mu_{C}}$ (center right), along with the covariance parameters of the banded covariance model $\phi_{S}$ (bottom). Only a subset of the $\phi$ 's are shown. 


\section{Inverse problem solution - different noise levels}
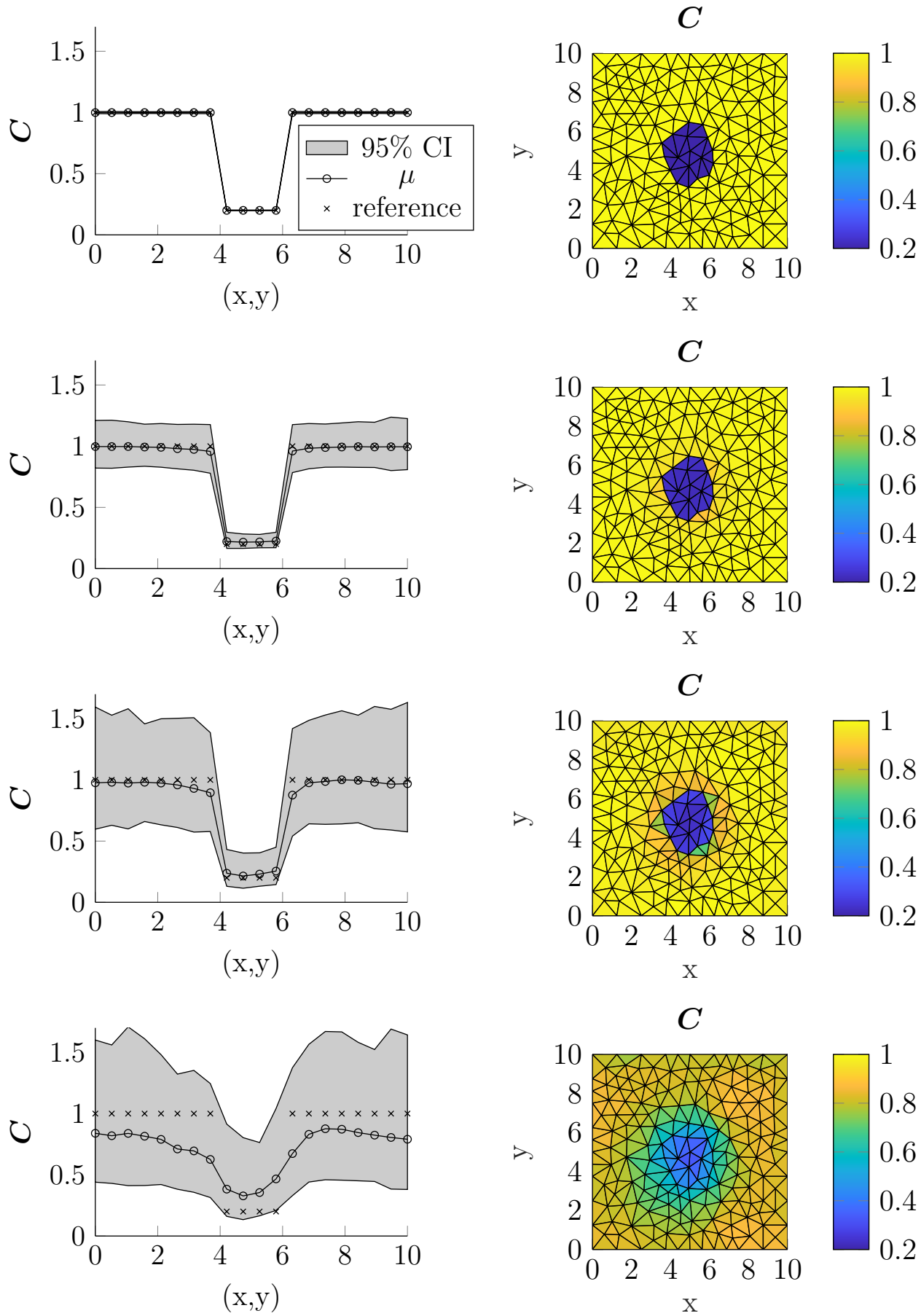

Figure 16: Inference results at different SNRs. The plots show the inferred means of the Young's moduli along the diagonal (left column) and over the whole domain (right column) for no noise (top row) as well as for $60 \mathrm{~dB}$ (2nd row), 50dB (3rd row) and 40dB (4th row) noise levels. 
inclusion. In particular, we employed the following stress-strain relations ${ }^{9}$ :

$$
\boldsymbol{\sigma}_{e}= \begin{cases}\boldsymbol{D} \boldsymbol{\epsilon}_{e} \odot \exp \left[10 \boldsymbol{\epsilon}_{e} \odot \boldsymbol{\epsilon}_{e}\right] & \text { if the element is in the inclusion, } \\ \boldsymbol{D} \boldsymbol{\epsilon}_{e}, & \text { otherwise }\end{cases}
$$

where $\odot$ denotes component-wise multiplication for vectors and the exponential is taken component-wise. Thus, the constitutive behavior of the material in the inclusion is nonlinear. This particular material model is motivated by [97] where it was used it to reconstruct nonlinear breast tissue properties in the context of elastography. A plot of the resulting stress-strain relation compared to the linear one is provided in Figure 17.

In order to assess the proposed method's ability to identify model errors, for the inverse problem, a linear elastic constitutive law is assumed (as in the previous subsection) with unknown Young's moduli. The model error parameters $\boldsymbol{\lambda}$ are assumed unknown and one per element is employed, i.e. 325 in total. In addition, we have 1668 latent variables which include, as in section 4.3 , stresses $\boldsymbol{\Sigma}$, (interior) displacements $\boldsymbol{U}_{i}$ as well as the material parameters $\boldsymbol{C}$. We recall from section 3 that two approximating densities $q$ and $r$ are needed with parameters $\boldsymbol{\phi}$ and $\boldsymbol{\xi}$ respectively, each of which is of dimension 226578 (i.e. 453156 in total).

In Figure 18, the learned $\boldsymbol{\lambda}$ 's are depicted as well as the evolution (of selected ones) over the EM iterations performed. As it can be clearly seen, for almost all the elements belonging to the inclusion, the algorithm correctly identifies the presence of model error and sets the corresponding precisions $\boldsymbol{\lambda}$ to very small values indicating large discrepancies between the true stresses and the ones predicted by the linear elastic constitutive law. In the same Figure we show the evolution of the ELBO objective $\mathcal{L}_{i n v}$. As discussed in section 3.2 there is no guarantee that $\mathcal{L}_{i n v}$ will be always ascending due to the second variational approximation employed for the normalization constant of the prior.

A comparison between the reference solution and the posterior means and variances for the stresses and displacements is provided in Figure 19. We note that outside of the inclusion, where the constitutive law is found to be adequate, the right material parameters (Young's moduli) are identified. For the elements over which model errors were identified (i.e. small $\boldsymbol{\lambda}$ 's) the material parameter values are essentially determined by the smoothing prior. Despite the untrustworthiness of the constitutive law in these elements, the model can still correctly identify the true, latent stresses as it can be seen in Figure 20, where the posterior means and credible intervals along the diagonal are depicted. For the stresses in the inclusion elements, it is effectively the (discretized) equations of equilibrium (i.e. the conservation law) that are the sole source of information, in combination with the stresses from the other elements which, in the presence of a reliable constitutive law, are correctly identified. It is

${ }^{9}$ The matrix $\boldsymbol{D}=\frac{1}{1-\nu^{2}}\left[\begin{array}{ccc}1 & \nu & 0 \\ \nu & 1 & 0 \\ 0 & 0 & \frac{1-\nu}{2}\end{array}\right]$ represents the standard stress-strain relation in plane stress when the Young's modulus is 1.0. 
therefore essential to correctly capture these correlations in the approximate posteriors. In the same Figure we also depict the posterior inference results when a diagonal covariance matrix is used for the approximating densities $q$ and $r$ (section 3). While the diagonal covariance model offers a much more efficient framework for inference and learning (due to the significantly lower number of optimization parameters), it ignores some of the aforementioned dependencies and can therefore lead to the wrong conclusions.

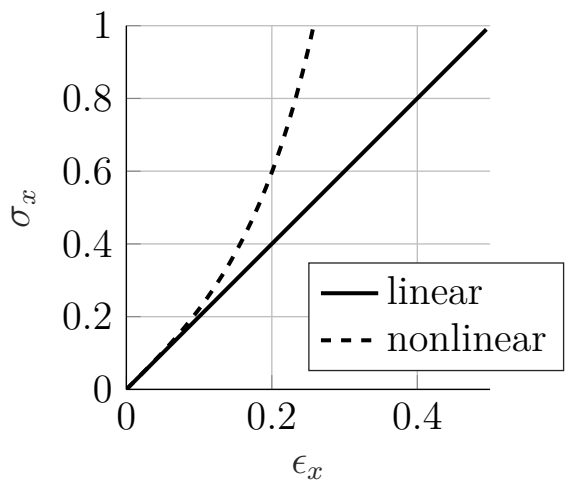

Figure 17: Employed nonlinear stress-strain relation (in one dimension) for the inclusion material. 

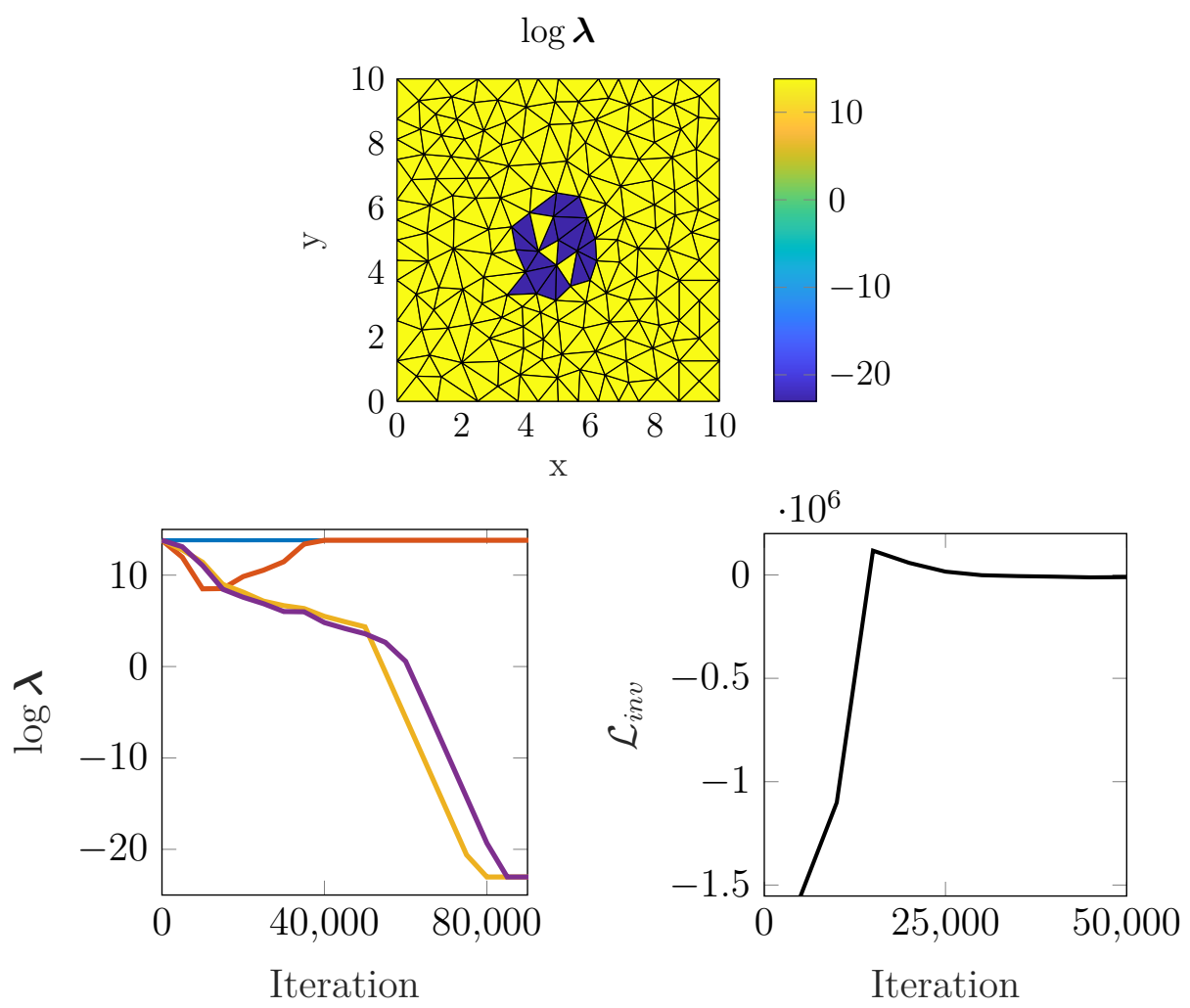

Figure 18: Learned values for parameters $\boldsymbol{\lambda}$ in log-scale over the whole domain (top), convergence behavior for some selected $\boldsymbol{\lambda}$ 's (lower left) and evolution of the objective function $\mathcal{L}_{i n v}$ over the number of iterations (lower right). 


\section{Model error estimation - inferred mean values, variances and reference}
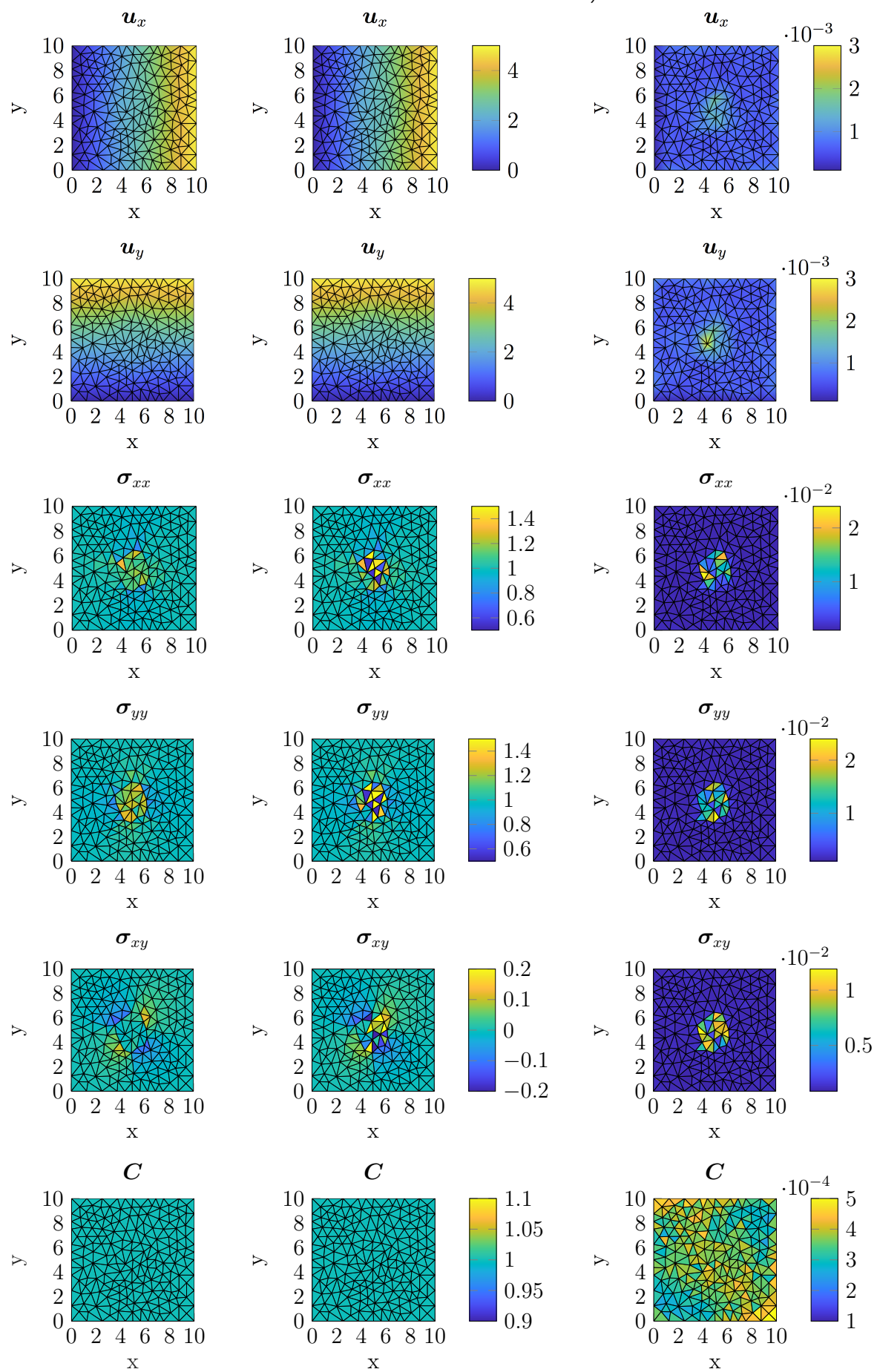

Figure 19: The plots compare the ground truth (left column), the inferred posterior means (center column) and variances (right column) of the displacements and stresses for the inverse problem with model error in section 4.4 . 

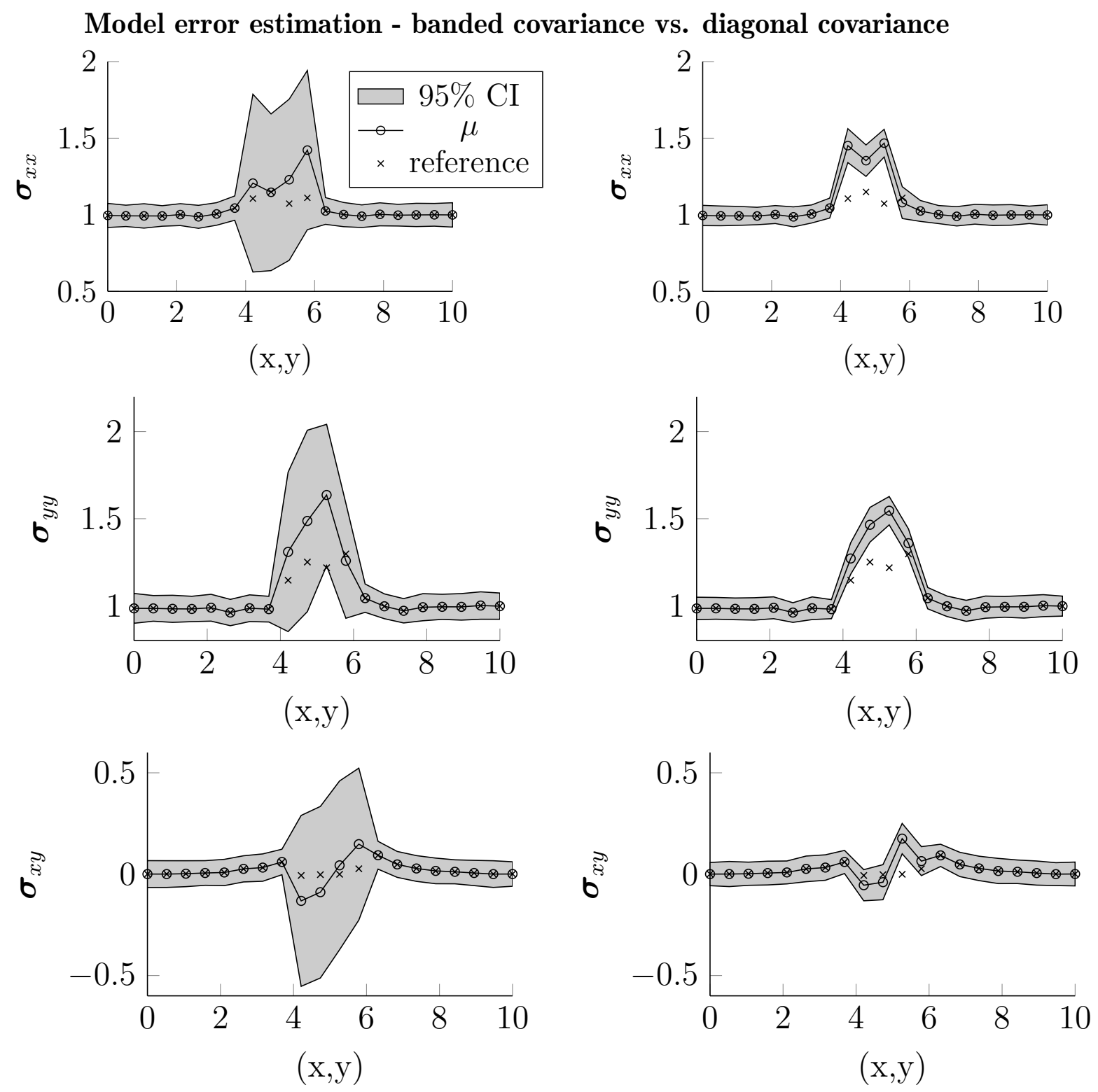

Figure 20: Comparison of the ground truth values along the diagonal with the inferred posterior means and $95 \%$ credible intervals for displacements and stresses. The results in the left column were obtained using a banded covariance and the results in the right column using a diagonal covariance model. 


\section{Conclusion}

We have presented a new formulation for model calibration and validation problems in the context of continuum thermodynamics. Rather than using associated forward models as black-boxes, we advocate making use of the governing equations as information sources which imply constraints for the state variables. We enforce these constraints probabilistically and quantify their validity in a Bayesian fashion. Reliable equations such as conservation laws imply stronger probabilistic constraints, whereas constitutive laws (or phenomenological closures in general) can be modeled hierarchically in order to infer their validity from the observational data. The formulation advocated employs an undirected probabilistic model which explicitly contains the dependencies between the state variables and can be used in place of the blackbox forward model. As a result the solution of, even ill-posed, deterministic forward problems is recast as a well-posed probabilistic inference task. More importantly, in the context of inverse problems it leads to an augmented posterior that distinguishes between the different error sources and can quantify model errors in a interpretable manner that respects known physical invariances.

One of the challenges associated with the proposed formulation pertains to the dimensionality of the densities involved. While these consists of large products of explicit terms, they involve all the physical state variables and as such live in much higher dimensions as compared to canonical Bayesian posteriors. We advocate a doublelayer Stochastic Variational Inference scheme that scales linearly with the number of optimization parameters and provides closed-form, approximate inference results but much more efficiently than Monte Carlo-based sampling schemes. We demonstrate the potential of this framework in the context of biomechanics where the solution of the aforementioned issues can significantly impact progress in the non-invasive, diagnostic capabilities and assist in the development of patient-specific treatment strategies.

Important extensions in increasing the efficacy of the proposed formulation relate to the representation of the latent variables and adaptivity. In this presentation, we have employed standard finite element basis functions to represent the physical fields. While this enables the use of pre-existing FE machinery, it does not necessarily provide the sparsest representation nor the one that minimizes discretization errors. We envision that tools from Dictionary Learning/Sparse Coding [80] could be valuable in this direction. We note also that the infinite dimensional governing equations potentially provide an infinite amount of data (e.g. as $N_{e} \rightarrow \infty$ in Equation (7) or $N_{c} \rightarrow \infty$ in Equation (9)) which open the door to very rich and expressive representations such as those implied by Deep Neural Nets [45, 99, 103]. Finally, we envision that significant scalability improvements can be achieved by an adaptive scheme which will initiate with a small number of $N_{e}$ and $N_{c}$ and progressively add weighting functions (Equation (7)) as well as interrogation points for the constitutive law (Equation (9)). This sequential enrichment of the prior/posterior model apart from providing a natural tempering effect, can be guided by posterior inferences. For example additional 
weighting functions of interrogation points $\boldsymbol{x}^{\left(i_{c}\right)}$ can be added in areas of the problem domain where the posterior variance of the state variables is largest. Several other metrics of the posterior uncertainty could be used as drivers of such adaptive schemes which will enable superior allocation of computational resources in regions that are most informative with regards to the posterior beliefs. 


\section{References}

[1] Arnold, A., Reichling, S., Bruhns, O., and Mosler, J. (2010). Efficient computation of the elastography inverse problem by combining variational mesh adaption and a clustering technique. PHYSICS IN MEDICINE AND BIOLOGY, 55(7):20352056.

[2] Attias, H. (2000). A Variational Bayesian Framework for Graphical Models. In In Advances in Neural Information Processing Systems 12, pages 209-215. MIT Press.

[3] Barbone, P. E., Rivas, C. E., Harari, I., Albocher, U., Oberai, A. A., and Zhang, Y. (2010). Adjoint-weighted variational formulation for the direct solution of inverse problems of general linear elasticity with full interior data. Int. J. Num. Meth. Eng., 81(13):1713-1736.

[4] Bardsley, J. (2013). Gaussian Markov random field priors for inverse problems. Inverse Problems and Imaging, 7(2):397-416.

[5] Bayarri, M., Berger, J., Paulo, R., Sacks, J., Cafeo, J., Cavendish, J., Lin, C., and Tu, J. (2007). A framework for validation of computer models. TECHNOMETRICS, 49(2):138 - 154 .

[6] Beal, M. J. (2003). Variational algorithms for approximate Bayesian inference. University of London United Kingdom.

[7] Berliner, L., Jezek, K., Cressie, N., Kim, Y., Lam, C., and van der Veen, C. (2008). Modeling dynamic controls on ice streams: a Bayesian statistical approach. Journal of Glaciology, 54(187):705-714.

[8] Biegler, L., Biros, G., Ghattas, O., Heinkenschloss, M., Keyes, D., Mallick, B., Tenorio, L., Waanders, B. v. B., Willcox, K., and Marzouk, Y., editors (2010). Large-Scale Inverse Problems and Quantification of Uncertainty. Wiley, Chichester, West Sussex, 1 edition edition.

[9] Bilionis, I. and Koutsourelakis, P. S. (2012). Free energy computations by minimization of Kullback-Leibler divergence: An efficient adaptive biasing potential method for sparse representations. Journal of Computational Physics, 231(9):3849 -3870 .

[10] Bilionis, I. and Zabaras, N. (2014). Solution of inverse problems with limited forward solver evaluations: a Bayesian perspective. Inverse Problems, 30(1):015004.

[11] Bishop, C. M. (2006). Pattern recognition and machine learning. Information science and statistics. Springer, New York.

[12] Bishop, C. M. and Tipping, M. E. (2000). Variational relevance vector machines. Proceedings of the Sixteenth conference on Uncertainty in artificial intelligence, (1):46-53. 
[13] Blei, D. M., Kucukelbir, A., and McAuliffe, J. D. (2017). Variational Inference: A Review for Statisticians. Journal of the American Statistical Association, 112(518):859-877.

[14] Brynjarsdottir, J. and O'Hagan, A. (2014). Learning about physical parameters: The importance of model discrepancy. Inverse Problems, 30(11):114007.

[15] Bui-Thanh, T. and Girolami, M. (2014). Solving large-scale PDE-constrained Bayesian inverse problems with Riemann manifold Hamiltonian Monte Carlo. Inverse Problems, 30(11):114014.

[16] Bui-Thanh, T., Willcox, K., and Ghattas, O. (2008). Model Reduction for LargeScale Systems with High-Dimensional Parametric Input Space. SIAM Journal on Scientific Computing, 30(6):3270.

[17] Candes, E., Romberg, J., and Tao, T. (2006). Robust uncertainty principles: Exact signal reconstruction from highly incomplete frequency information. IEEE Trans. Information Theory, 52:489-509.

[18] Carvalho, C. M., Polson, N. G., and Scott, J. G. (2009). Handling sparsity via the horseshoe. In International Conference on Artificial Intelligence and Statistics, pages $73-80$.

[19] Chkrebtii, O. A., Campbell, D. A., Calderhead, B., and Girolami, M. A. (2016). Bayesian Solution Uncertainty Quantification for Differential Equations. Bayesian Analysis, 11(4):1239-1267.

[20] Chopin, N., Lelièvre, T., and Stoltz, G. (2012). Free Energy Methods for Bayesian Inference: Efficient Exploration of Univariate Gaussian Mixture Posteriors. Statistics and Computing, 22(4):897-916.

[21] Cockayne, J., Oates, C., Sullivan, T., and Girolami, M. (2016). Probabilistic Numerical Methods for Partial Differential Equations and Bayesian Inverse Problems. arXiv:1605.07811 [cs, math, stat].

[22] Cockayne, J., Oates, C., Sullivan, T., and Girolami, M. (2017). Bayesian Probabilistic Numerical Methods. arXiv:1702.03673 [cs, math, stat].

[23] Cover, T. and Thomas, J. (1991). Elements of Information Theory. John Wiley $\&$ Sons.

[24] Cui, T., Law, K. J., and Marzouk, Y. M. (2016). Dimension-independent likelihood-informed MCMC. Journal of Computational Physics, 304:109-137.

[25] Cui, T., Martin, J., Marzouk, Y. M., Solonen, A., and Spantini, A. (2014). Likelihood-informed dimension reduction for nonlinear inverse problems. Inverse Problems, 30(11):114015.

[26] Cui, T., Marzouk, Y. M., and Willcox, K. E. (2015). Scalable posterior approximations for large-scale Bayesian inverse problems via likelihood-informed parameter and state reduction. arXiv preprint arXiv:1510.06053. 
[27] Cullen, M., Freitag, M. A., Kindermann, S., and Scheichl, R. (2013). Large Scale Inverse Problems, Computational Methods and Applications in the Earth Sciences. De Gruyter, Berlin, Boston.

[28] Curtis, C. and et al. (2012). The genomic and transcriptomic architecture of 2,000 breast tumours reveals novel subgroups. Nature.

[29] Del Moral, P., Doucet, A., and Jasra, A. (2012). On adaptive resampling strategies for sequential Monte Carlo methods. Bernoulli, 18(1):252-278.

[30] Della Pietra, S., Della Pietra, V., and Lafferty, J. (1997). Inducing Features of Random Fields. IEEE Transactions on Pattern Analysis and Machine Intelligence, 19(4):380-393.

[31] Dempster, A. A., Laird, N. N., and Rubin, D. D. B. (1977). Maximum likelihood from incomplete data via the EM algorithm. Journal of the Royal Statistical Society Series B Methodological, 39(1):1-38.

[32] Diaconis, P. (1988). Bayesian numerical analysis. Statistical decision theory and related topics $I V, 1: 163-175$.

[33] Dodwell, T. J., Ketelsen, C., Scheichl, R., and Teckentrup, A. L. (2015). A Hierarchical Multilevel Markov Chain Monte Carlo Algorithm with Applications to Uncertainty Quantification in Subsurface Flow. SIAM/ASA Journal on Uncertainty Quantification, 3(1):1075-1108.

[34] Donoho, D. (2006). Compressed sensing. IEEE Transactions Information Theory, 52(4):1289-1306.

[35] Doyley, M., Srinivasan, S., Dimidenko, E., Soni, N., and Ophir, J. (2006). Enhancing the performance of model-based elastography by incorporating additional a priori information in the modulus image reconstruction process. PHYSICS IN MEDICINE AND BIOLOGY, 51(1):95-112.

[36] Doyley, M. M. (2012). Model-based elastography: a survey of approaches to the inverse elasticity problem. Physics in Medicine and Biology, 57(3):R35.

[37] Draper, D. (1995). Assessment and Propagation of Model Uncertainty. Journal of the Royal Statistical Society Series B-Methodological, 57(1):45-97.

[38] Ellam, L., Zabaras, N., and Girolami, M. (2016). A Bayesian approach to multiscale inverse problems with on-the-fly scale determination. Journal of Computational Physics, 326:115-140.

[39] Finlayson, B., editor (1972). The method of weighted residuals and variational principles, with application in fluid mechanics, heat and mass transfer, Volume $8 \%$. Academic Press, New York.

[40] Flath, H. P., Wilcox, L. C., Akçelik, V., Hill, J., van Bloemen Waanders, B., and Ghattas, O. (2011). Fast Algorithms for Bayesian Uncertainty Quantifica- 
tion in Large-Scale Linear Inverse Problems Based on Low-Rank Partial Hessian Approximations. SIAM J. Sci. Comput., 33(1):407-432.

[41] Franck, I. M. and Koutsourelakis, P. (2016). Sparse Variational Bayesian approximations for nonlinear inverse problems: Applications in nonlinear elastography. Computer Methods in Applied Mechanics and Engineering, 299:215-244.

[42] Ganne-Carrié, N., Ziol, M., de Ledinghen, V., Douvin, C., Marcellin, P., Castera, L., Dhumeaux, D., Trinchet, J., and Beaugrand, M. (2006). Accuracy of liver stiffness measurement for the diagnosis of cirrhosis in patients with chronic liver diseases. Hepatology, 44(6):1511-1517.

[43] Girolami, M. and Calderhead, B. (2011). Riemann manifold Langevin and Hamiltonian Monte Carlo methods. Journal of the Royal Statistical Society: Series B (Statistical Methodology), 73(2):123-214.

[44] Gockenbach, M. S. and Khan, A. A. (2005). Identification of lame parameters in linear elasticity: A fixed point approach. JOURNAL OF INDUSTRIAL AND MANAGEMENT OPTIMIZATION, 1(4):487-497.

[45] Goodfellow, I. J., Bengio, Y., and Courville, A. C. (2016). Deep Learning. Adaptive computation and machine learning. MIT Press.

[46] Green, P. J., Latuszynski, K., Pereyra, M., and Robert, C. P. (2015). Bayesian computation: a summary of the current state, and samples backwards and forwards. Statistics and Computing, 25(4):835-862.

[47] Hennig, P. and Hauberg, S. (2014). Probabilistic Solutions to Differential Equations and their Application to Riemannian Statistics. In Proc. of the 17th int. Conf. on Artificial Intelligence and Statistics (AISTATS), volume 33. JMLR, W\&CP.

[48] Hennig, P., Osborne, M. A., and Girolami, M. (2015). Probabilistic numerics and uncertainty in computations. Proceedings. Mathematical, physical, and engineering sciences / the Royal Society, 471(2179):20150142.

[49] Higdon, D., Gattiker, J., Williams, B., and Rightley, M. (2008). Computer model calibration using high-dimensional output. Journal of the American Statistical Association, 103(482):570-583.

[50] Higdon, D., Kennedy, M., Cavendish, J., Cafeo, J., and Ryne, R. (2004). Combining Field Data and Computer Simulations for Calibration and Prediction. SIAM Journal on Scientific Computing, 26(2):448-466.

[51] Hoffman, M. D., Blei, D. M., Wang, C., and Paisley, J. (2013). Stochastic variational inference. The Journal of Machine Learning Research, 14(1):13031347.

[52] Holloman, C., Lee, H., and Higdon, D. (2006). Multi-resolution Genetic Algorithms and Markov Chain Monte Carlo. Journal of Computational and Graphical Statistics, pages 861-879. 
[53] Hughes, T. J. R. (2000). The Finite Element Method-Linear Static and Dynamic Finite Element Analysis. Dover.

[54] Ishwaran, H. and Rao, J. S. (2005). Spike and slab variable selection: Frequentist and Bayesian strategies. The Annals of Statistics, 33(2):730-773.

[55] Jordan, M. I., Ghahramani, Z., Jaakkola, T. S., and Saul, L. K. (1999). An Introduction to Variational Methods for Graphical Models. Mach. Learn., 37(2):183233.

[56] Kass, R. E. and Raftery, A. E. (1995). Bayes Factors. Journal of the American Statistical Association, 90(430):773-795.

[57] Kennedy, M. C. and O'Hagan, A. (2001). Bayesian calibration of computer models. Journal Of The Royal Statistical Society Series B-Statistical Methodology, 63:425-450.

[58] Khalil, A. S., Chan, R. C., Chau, A. H., Bouma, B. E., and Mofrad, M. R. K. (2005). Tissue elasticity estimation with optical coherence elastography: toward mechanical characterization of in vivo soft tissue. Annals of Biomedical Engineering, 33(11):1631-1639.

[59] Kingma, D. and Ba, J. (2014). Adam: A Method for Stochastic Optimization. arXiv:1412.6980 [cs], pages 1-15.

[60] Kingma, D. P. and Welling, M. (2013). Auto-Encoding Variational Bayes. $(\mathrm{Ml}): 1-14$.

[61] Koller, D. and Friedman, N. (2009). Probabilistic graphical models: principles and techniques. Adaptive computation and machine learning. MIT Press, Cambridge, MA.

[62] Koutsourelakis, P. (2009). A multi-resolution, non-parametric, Bayesian framework for identification of spatially-varying model parameters. Journal of Computational Physics, 228(17):6184-6211.

[63] Koutsourelakis, P.-S. (2012). A novel Bayesian strategy for the identification of spatially varying material properties and model validation: an application to static elastography. International Journal for Numerical Methods in Engineering, 91(3):249-268.

[64] Lan, S., Bui-Thanh, T., Christie, M., and Girolami, M. (2016). Emulation of higher-order tensors in manifold Monte Carlo methods for Bayesian Inverse Problems. Journal of Computational Physics, 308:81-101.

[65] Lee, H., Higdon, D., Bi, Z., Ferreira, M., and West, M. (2002). Markov random field models for high-dimensional parameters in simulations of fluid flow in porous media. TECHNOMETRICS, 44(3):230 - 241.

[66] Lewicki, M. and Sejnowski, T. J. (2000). Learning overcomplete representations. Neural Computation, 12(2):337-365. 
[67] MacKay, D. J. (2003). Information theory, inference and learning algorithms. Cambridge university press.

[68] Martin, J., Wilcox, L. C., Burstedde, C., and Ghattas, O. (2012). A stochastic Newton MCMC method for large-scale statistical inverse problems with application to seismic inversion. SIAM Journal on Scientific Computing, 34(3):A1460-A1487.

[69] Marzouk, Y. M., Najm, H. N., and Rahn, L. A. (2007). Stochastic spectral methods for efficient Bayesian solution of inverse problems. J. Comput. Phys, $224(2): 560-586$.

[70] Mattingly, J. C., Pillai, N. S., and Stuart, A. M. (2012). Diffusion limits of the random walk Metropolis algorithm in high dimensions. The Annals of Applied Probability, 22(3):881-930.

[71] Moral, P. D., Doucet, A., and Jasra, A. (2006). Sequential Monte Carlo for Bayesian computation (with discussion). In Bayesian Statistics 8. Oxford University Press.

[72] Moser, R. D. and Oliver, T. A. (2015). Validation of Physical Models in the Presence of Uncertainty. In Ghanem, R., Higdon, D., and Owhadi, H., editors, Handbook of Uncertainty Quantification, pages 1-28. Springer International Publishing.

[73] Murray, I. and Ghahramani, Z. (2004). Bayesian Learning in Undirected Graphical Models: Approximate MCMC Methods.

[74] Muthupillai, R., Lomas, D., Rossman, P., Greenleaf, J., Manduca, A., and Ehman, R. (1995). Magnetic resonance elastography by direct visualization of propagating acoustic strain waves. Science, 269(5232):1854-1857.

[75] Neal, R. and Hinton, G. E. (1998). A View Of The Em Algorithm That Justifies Incremental, Sparse, And Other Variants. In Learning in Graphical Models, pages 355-368. Kluwer Academic Publishers.

[76] Oberai, A., Gokhale, N., Doyley, M., and Bamber, J. (2004a). Evaluation of the adjoint equation based algorithm for elasticity imaging. PHYSICS IN MEDICINE AND BIOLOGY, 49(13):2955-2974.

[77] Oberai, A., Gokhale, N., Doyley, M., and Bamber, J. (2004b). Evaluation of the adjoint equation based algorithm for elasticity imaging. PHYSICS IN MEDICINE AND BIOLOGY, 49(13):2955-2974.

[78] Oberai, A. A., Gokhale, N. H., Goenezen, S., Barbone, P. E., Hall, T. J., Sommer, A. M., and Jiang, J. (2009). Linear and nonlinear elasticity imaging of soft tissue in vivo: demonstration of feasibility. PHYSICS IN MEDICINE AND BIOLOGY, 54(5):1191-1207.

[79] O’Hagan, A., Kennedy, M., and Oakley, J. E. (1999). Uncertainty analysis and other inference tools for complex computer codes (with discussion). In Bernardo, J. and al, e., editors, In Bayesian Statistics 6. Oxford University Press. 
[80] Olshausen, B. A. and Field, D. J. (1997). Sparse coding with an overcomplete basis set: A strategy employed by V1? Vision Research, 37(23):3311-3325.

[81] Olson, L. G. and Throne, R. D. (2010). Numerical simulation of an inverse method for tumour size and location estimation. Inv. Prob. Sc. Eng., 18(6):813834.

[82] Ophir, J., Cespedes, I., Ponnekanti, H., Yazdi, Y., and Li, X. (1991). Elastography - a quantitative method for imaging the elasticity of biological tissues. ULTRASONIC IMAGING, 13(2):111 - 134.

[83] Paisley, J., Blei, D., and Jordan, M. (2012). Variational Bayesian Inference with Stochastic Search. Icml, (2000):1367-1374.

[84] Petra, N., Martin, J., Stadler, G., and Ghattas, O. (2014). A Computational Framework for Infinite-Dimensional Bayesian Inverse Problems, Part II: Stochastic Newton MCMC with Application to Ice Sheet Flow Inverse Problems. SIAM Journal on Scientific Computing, 36(4):A1525-A1555.

[85] Pillai, N. S., Stuart, A. M., and Thiéry, A. H. (2012). Optimal scaling and diffusion limits for the Langevin algorithm in high dimensions. The Annals of Applied Probability, 22(6):2320-2356.

[86] Rezende, D. J., Mohamed, S., and Wierstra, D. (2014). Stochastic backpropagation and approximate inference in deep generative models. Proceedings of The 31st ldots, 32:1278-1286.

[87] Robbins, H. and Monro, S. (1951). A Stochastic Approximation Method. The Annals of Mathematical Statistics, 22(3):400-407.

[88] Roberts, G. O. and Rosenthal, J. S. (1998). Optimal scaling of discrete approximations to Langevin diffusions. Journal of the Royal Statistical Society: Series B (Statistical Methodology), 60(1):255-268.

[89] Roberts, G. O. and Tweedie, R. L. (1996). Exponential convergence of Langevin diffusions and their discrete approximations. Bernoulli, 2:341-364.

[90] Rosic, B. V., Litvinenko, A., Pajonk, O., and Matthies, H. G. (2012). Samplingfree linear Bayesian update of polynomial chaos representations. J. Comput. Physics, 231(17):5761-5787.

[91] Sargsyan, K., Najm, H. N., and Ghanem, R. (2015). On the Statistical Calibration of Physical Models. International Journal of Chemical Kinetics, 47(4):246-276.

[92] Sarvazyan, A. and Hall, T., editors (2011). Elasticity Imaging Part I \& II, volume 7,8. Cur. Med. Imag. Rev.

[93] Silva, R. and Ghahramani, Z. (2006). Bayesian Inference for \{G\}aussian Mixed Graph Models. Uai.

[94] Silva, R. and Ghahramani, Z. (2009). The Hidden Life of Latent Variables 
: Bayesian Learning with Mixed Graph Models. Journal of Machine Learning Research, 10:1187-1238.

[95] Spantini, A., Solonen, A., Cui, T., Martin, J., Tenorio, L., and Marzouk, Y. (2015). Optimal Low-rank Approximations of Bayesian Linear Inverse Problems. SIAM Journal on Scientific Computing, 37(6):A2451-A2487.

[96] Strong, M. and Oakley, J. (2014). When Is a Model Good Enough? Deriving the Expected Value of Model Improvement via Specifying Internal Model Discrepancies. SIAM/ASA Journal on Uncertainty Quantification, 2(1):106-125.

[97] Sun, L. Z., Wang, Z. G., Liu, Y., and Wang, G. (2009). Elastography method for reconstruction of nonlinear breast tissue properties. International Journal of Biomedical Imaging, 2009.

[98] Titsias, M. K. and Lázaro-Gredilla, M. (2014). Doubly stochastic variational Bayes for non-conjugate inference. ICML.

[99] Tripathy, R. and Bilionis, I. (2018). Deep UQ: Learning deep neural network surrogate models for high dimensional uncertainty quantification. arXiv:1802.00850 [physics, stat].

[100] Wainwright, M. and Jordan, M. (2008). Graphical models, exponential families, and variational inference. In Foundations and Trends in Machine Learning, volume 1, pages $1-305$.

[101] Wainwright, M. J., Jaakkola, T. S., and Willsky, A. S. (2002). A new class of upper bounds on the log partition function. In Proceedings of the Eighteenth conference on Uncertainty in artificial intelligence, pages 536-543. Morgan Kaufmann Publishers Inc.

[102] Wipf, D. P. and Nagarajan, S. S. (2008). A New View of Automatic Relevance Determination. In Platt, J. C., Koller, D., Singer, Y., and Roweis, S. T., editors, Advances in Neural Information Processing Systems 20, pages 1625-1632. Curran Associates, Inc.

[103] Zhu, Y. and Zabaras, N. (2018). Bayesian Deep Convolutional EncoderDecoder Networks for Surrogate Modeling and Uncertainty Quantification. arXiv:1801.06879 [physics, stat]. 


\section{A Appendix}

This Appendix contains details for the derivatives of the ELBO objectives formulated in section 3 for the solution of forward and inverse problems under the framework proposed.

\section{A.1 Derivatives of $\mathcal{L}_{\text {for }}$}

We recall from Equation (36) that:

$$
\begin{aligned}
\mathcal{L}_{f o r}(\boldsymbol{\phi})= & \mathbb{E}_{q(\boldsymbol{y} ; \phi)}\left[\log \psi_{1}(\boldsymbol{\sigma})\right]+\mathbb{E}_{q(\boldsymbol{y} ; \boldsymbol{\phi})}\left[\log \psi_{2}\left(\boldsymbol{\sigma}, \boldsymbol{u}_{i}, \boldsymbol{C}=\boldsymbol{c} ; \boldsymbol{\lambda}\right)\right] \\
& +\mathbb{E}_{q(\boldsymbol{y} ; \boldsymbol{\phi})}\left[\log \psi_{4}(\boldsymbol{\sigma})\right]+\mathbb{E}_{q(\boldsymbol{y} ; \boldsymbol{\phi})}\left[\log \psi_{5}\left(\boldsymbol{u}_{i}\right)\right]+\mathbb{H}[q(\boldsymbol{y} ; \boldsymbol{\phi})]
\end{aligned}
$$

where $q(\boldsymbol{y} ; \phi)=\mathcal{N}\left(\boldsymbol{y} \mid \mathbf{0}, \boldsymbol{S}=\boldsymbol{L} \boldsymbol{L}^{T}\right)$ and $\boldsymbol{\phi}=\{\boldsymbol{\mu}, \boldsymbol{L}\}$. As discussed in section 3.4 derivatives with respect to $\boldsymbol{\phi}$ of all the terms involving expectations with respect to $q$ are computed with Monte Carlo using reparameterization trick (see Equation (41) and Equation (42)). As for the entropy term (up to a constant):

$$
\mathbb{H}[q(\boldsymbol{y} ; \boldsymbol{\phi})]=-\frac{1}{2} \log |\boldsymbol{S}|-\frac{1}{2} \operatorname{dim}(\boldsymbol{y})=-\log |\boldsymbol{L}|
$$

Hence:

$$
\nabla_{\boldsymbol{u}} \mathbb{H}[q(\boldsymbol{y} ; \boldsymbol{\phi})]=0, \quad \nabla_{\boldsymbol{L}} \mathbb{H}[q(\boldsymbol{y} ; \boldsymbol{\phi})]=-\boldsymbol{L}^{-1}
$$

\section{A.2 Derivatives of $\mathcal{L}_{i n v}$}

We recall from Equation (40) that:

$$
\begin{aligned}
\mathcal{L}_{i n v}= & \mathbb{E}_{q(\boldsymbol{y} ; \phi)}\left[\log p\left(\boldsymbol{u}_{o b s} \mid \boldsymbol{U}_{i}\right)\right]+\mathbb{H}[q(\boldsymbol{y} ; \boldsymbol{\phi})] \\
& +\mathbb{E}_{q(\boldsymbol{y} ; \boldsymbol{\phi})}\left[\log \pi_{\boldsymbol{\lambda}}\left(\boldsymbol{\sigma}, \boldsymbol{c}, \boldsymbol{u}_{i}, \boldsymbol{U}_{b}=\boldsymbol{u}_{0}\right)\right]-\hat{\mathcal{F}}_{i n v}(r(\boldsymbol{y} ; \boldsymbol{\xi}), \boldsymbol{\lambda}) \\
& +\log p(\boldsymbol{\lambda})
\end{aligned}
$$

where $\hat{\mathcal{F}}_{\text {inv }}(r(\boldsymbol{y} ; \boldsymbol{\xi}), \boldsymbol{\lambda})$ is given in Equation (39) as:

$$
\hat{\mathcal{F}}_{i n v}(r(\boldsymbol{y} ; \boldsymbol{\xi}), \boldsymbol{\lambda})=\mathbb{E}_{r(\boldsymbol{y} ; \boldsymbol{\xi})}\left[\log \pi_{\boldsymbol{\lambda}}\left(\boldsymbol{y}, \boldsymbol{U}_{b}=\boldsymbol{u}_{0}\right)\right]+\mathbb{H}[r(\boldsymbol{y} ; \boldsymbol{\xi})]
$$

Both $q(\boldsymbol{y} ; \phi)$ and $r(\boldsymbol{y} ; \boldsymbol{\xi}), \boldsymbol{\lambda})$ are multivariate Gaussian with banded covariance represented by $\boldsymbol{\phi}$ and $\boldsymbol{\xi}$ respectively. Derivatives of terms involving expectations with respect to $q$ or $r$ are needed in the E-step and are computed with Monte Carlo and the reparametrization trick. The entropy terms and their derivatives can be computed as in Equation (47) and Equation (48). 
For the M-step, derivatives of the objective $\mathcal{L}_{i n v}$ with respect to $\boldsymbol{\lambda}$ are needed. We note that these consist of three terms:

$$
\begin{aligned}
\frac{\partial \mathcal{L}_{i n v}}{\partial \boldsymbol{\lambda}}= & \mathbb{E}_{q(\boldsymbol{y} ; \boldsymbol{\phi})}\left[\frac{\partial \log \pi_{\boldsymbol{\lambda}}\left(\boldsymbol{\sigma}, \boldsymbol{c}, \boldsymbol{u}_{i}, \boldsymbol{U}_{b}=\boldsymbol{u}_{0}\right)}{\partial \boldsymbol{\lambda}}\right]-\frac{\partial \hat{\mathcal{F}}_{i n v}(r(\boldsymbol{y} ; \boldsymbol{\xi}), \boldsymbol{\lambda})}{\partial \boldsymbol{\lambda}}-\frac{\partial \log p(\boldsymbol{\lambda})}{\partial \boldsymbol{\lambda}} \\
= & \mathbb{E}_{q(\boldsymbol{y} ; \boldsymbol{\phi})}\left[\frac{\partial \log \pi_{\boldsymbol{\lambda}}\left(\boldsymbol{\sigma}, \boldsymbol{c}, \boldsymbol{u}_{i}, \boldsymbol{U}_{b}=\boldsymbol{u}_{0}\right)}{\partial \boldsymbol{\lambda}}\right] \\
& -\mathbb{E}_{r(\boldsymbol{y} ; \boldsymbol{\xi})}\left[\frac{\partial \log \pi_{\boldsymbol{\lambda}}\left(\boldsymbol{\sigma}, \boldsymbol{c}, \boldsymbol{u}_{i}, \boldsymbol{U}_{b}=\boldsymbol{u}_{0}\right)}{\partial \boldsymbol{\lambda}}\right] \quad(\text { from Equation (39)) } \\
& +\frac{\partial \log p(\boldsymbol{\lambda})}{\partial \boldsymbol{\lambda}}
\end{aligned}
$$

From Equation (27) and Equation (24), we have:

$$
\frac{\partial \log \pi_{\boldsymbol{\lambda}}\left(\boldsymbol{\sigma}, \boldsymbol{c}, \boldsymbol{u}_{i}, \boldsymbol{U}_{b}=\boldsymbol{u}_{0}\right)}{\partial \lambda_{e}}=-\frac{1}{2}\left\|\boldsymbol{\sigma}_{e}-\boldsymbol{c}_{e} \boldsymbol{B}_{e} \boldsymbol{u}_{e}\right\|^{2}
$$

which leads to (in combination with Equation (30)) to:

$$
\begin{aligned}
\frac{\partial \mathcal{L}_{i n v}}{\partial \lambda_{e}}= & -\frac{1}{2} \mathbb{E}_{q(\boldsymbol{y} ; \boldsymbol{\phi})}\left[\left\|\boldsymbol{\sigma}_{e}-\boldsymbol{c}_{e} \boldsymbol{B}_{e} \boldsymbol{u}_{e}\right\|^{2}\right]+\frac{1}{2} \mathbb{E}_{r(\boldsymbol{y} ; \boldsymbol{\xi})}\left[\left\|\boldsymbol{\sigma}_{e}-\boldsymbol{c}_{e} \boldsymbol{B}_{e} \boldsymbol{u}_{e}\right\|^{2}\right] \\
& +\underbrace{\frac{\alpha_{0}-1}{\lambda_{e}}-\beta_{0}}_{\frac{\partial \log p(\boldsymbol{\lambda})}{\partial \lambda_{e}}}
\end{aligned}
$$

The first two terms are computed with Monte Carlo and the reparametrization trick.

\section{A.3 Parameter update using Adam}

Parameter updates for stochastic optimization are performed as

$$
\begin{aligned}
\boldsymbol{\phi}^{(k+1)} & =\boldsymbol{\phi}^{(k)}+\rho_{\phi}^{(k)} \nabla_{\phi} \mathcal{L}\left(\phi^{(k)}, \boldsymbol{\xi}^{(k)}, \boldsymbol{\lambda}^{(k)}\right), \\
\boldsymbol{\xi}^{(k+1)} & =\boldsymbol{\xi}^{(k)}-\rho_{\boldsymbol{\xi}}^{(k)} \nabla_{\boldsymbol{\xi}} \mathcal{L}\left(\boldsymbol{\phi}^{(k)}, \boldsymbol{\xi}^{(k)}, \boldsymbol{\lambda}^{(k)}\right), \\
\boldsymbol{\lambda}^{(k+1)} & =\boldsymbol{\lambda}^{(k)}+\rho_{\boldsymbol{\lambda}}^{(k)} \nabla_{\boldsymbol{\lambda}} \mathcal{L}\left(\boldsymbol{\phi}^{(k)}, \boldsymbol{\xi}^{(k)}, \boldsymbol{\lambda}^{(k)}\right),
\end{aligned}
$$

where $k \in \mathbb{N}_{0}$ and $\rho_{\xi}^{(k)}, \rho_{\phi}^{(k)}, \rho_{\lambda}^{(k)}$ are the respective step sizes at iteration $k$. According to the Robbins-Monro criteria [87], these update rules guarantee convergence to a local optimum, if the step size sequences satisfy the conditions

$$
\sum_{k=0}^{\infty} \rho^{(k)}=\infty \text { and } \quad \sum_{k=0}^{\infty} \rho^{(k)^{2}}<\infty
$$


For our purposes, we employ the update scheme ADAM proposed by [59]. It features an adaptive moment estimation for faster convergence by calculating exponentially decaying averages of the first and second moments of the gradient components $g_{j}$

$$
\begin{aligned}
m_{j}^{(k)} & =\beta_{1} m_{j}^{(k-1)}+\left(1-\beta_{1}\right) g_{j}^{(k)}, \\
v_{j}^{(k)} & =\beta_{2} v_{j}^{(k-1)}+\left(1-\beta_{2}\right) g_{j}^{(k)^{2}},
\end{aligned}
$$

with hyperparameters $\beta_{1}, \beta_{2}$ and the moment estimates initialized to $m_{j}^{(0)}=0$ and $v_{j}^{(0)}=0$, respectively. Bias correction is applied as

$$
\hat{m}_{j}^{(k)}=\frac{m_{j}^{(k)}}{1-\beta_{1}^{k}} \quad \text { and } \quad \hat{v}_{j}^{(k)}=\frac{v_{j}^{(k)}}{1-\beta_{2}^{k}},
$$

resulting in the component-wise parameter update rule

$$
\phi_{j}^{(k+1)}=\phi_{j}^{(k)}+\eta \frac{\hat{m}_{j}^{(k)}}{\sqrt{\hat{v}_{j}^{(k)}}+\varepsilon} .
$$

Hyperparameters need to be chosen problem dependent. We set them to $\eta=0.001$, $\beta_{1}=0.6, \beta_{2}=0.6$ and $\varepsilon=1 e^{-8}$ in all our experiments. 